C-type lectin receptors (CLRs). A large family of receptors that binds to carbohydrates through one or more carbohydrate-recognition domains. These receptors are expressed by many immune cells, including macrophages and dendritic cells, and are crucial for the detection of bacteria, viruses, helminth parasites, protozoa and fungi.
'Department of Microbiology and Immunobiology, Harvard Medical School, Boston, Massachusetts 02115, USA. ${ }^{2}$ Department of Microbiology, The University of Chicago, Chicago, Illinois 60637, USA. Correspondence to M.U.G. mgack@uchicago.edu Published online 13 May 2016

\title{
Viral evasion of intracellular DNA and RNA sensing
}

\section{Ying Kai Chan ${ }^{1}$ and Michaela U. Gack}

Abstract | The co-evolution of viruses with their hosts has led to the emergence of viral pathogens that are adept at evading or actively suppressing host immunity. Pattern recognition receptors (PRRs) are key components of antiviral immunity that detect conserved molecular features of viral pathogens and initiate signalling that results in the expression of antiviral genes. In this Review, we discuss the strategies that viruses use to escape immune surveillance by key intracellular sensors of viral RNA or DNA, with a focus on RIG-I-like receptors (RLRs), cyclic GMP-AMP synthase (cGAS) and interferon- $\gamma$ (IFN $\gamma)$-inducible protein 16 (IFI16). Such viral strategies include the sequestration or modification of viral nucleic acids, interference with specific post-translational modifications of PRRs or their adaptor proteins, the degradation or cleavage of PRRs or their adaptors, and the sequestration or relocalization of PRRs. An understanding of viral immune-evasion mechanisms at the molecular level may guide the development of vaccines and antivirals.

Despite rapid advances in the development of antiviral drugs for some viruses, such as HIV and hepatitis $\mathrm{C}$ virus (HCV), there remains an alarming paucity of antiviral drugs for many other clinically important viral pathogens. The continued emergence of new and previously known viral pathogens, as well as drug-resistant variants, highlights the urgent need for the development of novel and more effective vaccines and antivirals to combat viruses and mitigate human disease.

As obligate intracellular parasites, viruses require the host cell machinery and resources to replicate and propagate. Mammalian cells have evolved elaborate defence mechanisms to detect and inhibit viral replication. In turn, this has led to the emergence of viruses that are adept at manipulating and subverting host immune responses, which leads to enhanced virus-induced pathogenesis. These human-pathogenic viruses continue to be a leading cause of morbidity and mortality worldwide, as exemplified by the swine flu pandemic caused by the H1N1 subtype of influenza A virus in 2009 and the Ebola virus (EBOV) outbreak in West Africa in 2014. Therefore, an understanding of how viruses evade or antagonize the host antiviral response at the molecular level could guide the design of live attenuated vaccines and the development of new antiviral drugs. Furthermore, detailed insights into the molecular mechanisms by which infected cells sense viral pathogens may enable us to use the immune system for antiviral therapeutics.

Immediately after viral infection, the innate immune response is initiated by germline-encoded molecules termed pattern recognition receptors (PRRs).
PRRs recognize conserved features of viruses and other microorganisms, known as pathogen-associated molecular patterns (PAMPs), which include microbial nucleic acids, proteins and carbohydrates. Generally, these innate immune sensors can be categorized into two major classes depending on their subcellular location. PRRs that recognize extracellular PAMPs are typically found on the plasma membrane or endosomal membranes, and include the Toll-like receptors (TLRs) and the $\mathrm{C}$-type lectin receptors (CLRs) ${ }^{1,2}$. In most cases, these membrane-bound PRRs are predominantly expressed in immune cells, such as macrophages and dendritic cells. By contrast, intracellular PRRs are found in the cytoplasm or nucleus of mammalian cells, and include the NOD-like receptors (NLRs; also known as nucleotidebinding domain (NBD) and leucine-rich repeat (LRR)containing proteins), RIG-Ilike receptors (RLRs), and a group of intracellular DNA sensors that includes cyclic GMP-AMP synthase (cGAS) and interferon- $\gamma$ (IFN $\gamma$ )inducible protein $16(\mathrm{IFI} 16)^{3}$. These intracellular sensors are widely or ubiquitously expressed, which enables the detection of viral pathogens after invasion of the cytoplasm and/or nucleus in almost all cell types in a mammalian host.

Following the recognition of PAMPs, PRRs initiate innate immune signalling through the hierarchical activation of a PRR family-specific adaptor protein (for example, mitochondrial antiviral signalling protein (MAVS), stimulator of IFN genes (STING) or MYD88), as well as a common set of well-studied serine/threonine kinases and transcription factors. PRR-mediated 
NOD-like receptors

(NLRS). Nucleotide-binding

oligomerization domain

(NOD)-like receptors are

intracellular sensors of

microbial pathogen-associated

molecular patterns (PAMPs) or

damage-associated molecular

patterns (DAMPs). They

contain a conserved NOD

motif and activate diverse

signalling processes, such as

autophagy, interferon

responses and activation of the

inflammasome.

RIG-I-like receptors

(RLRs). Cytoplasmic RNA

helicases that function as viral

RNA sensors (such as retinoic acid-inducible gene I protein

(RIG-I) and melanoma differentiation-associated protein 5

(MDA5)) or innate regulatory molecules (such as LGP2).

RLRs have a central

DExD/H-box helicase domain

and a carboxy-terminal

domain, both of which are

required for RNA binding; in

addition, RIG-I and MDA5 have

a tandem caspase activation

and recruitment domain

(CARD) module for initiating

innate immune signal

transduction.

Cyclic GMP-AMP synthase

(cGAS). A cytosolic DNA sensor that, when activated, produces

the second messenger cyclic GMP-AMP (CGAMP), which in

turn activates stimulator of interferon genes (STING) to

induce the production of interferons. CCAS is crucial for the detection of DNA viruses and also retroviruses, which produce DNA through reverse transcription of their RNA genomes

\section{IFI16}

(Interferon- $\gamma$ (IFN $\gamma$ )-inducible protein 16). A pyrin and HIN200 domain-containing (PYHIN) protein family member that detects viral DNA inside the infected host cell and

initiates signalling through the adaptor protein stimulator of IFN genes (STING) to induce the expression of IFN genes. IFI1 6 can be found both in the nucleus and the cytoplasm of mammalian cells. signal transduction ultimately leads to the production of numerous host defence molecules, including type I and type III IFNs, and other pro-inflammatory cytokines and chemokines. Secreted IFNs are able to signal through their receptors in an autocrine and paracrine fashion to induce the expression of hundreds of IFNstimulated genes (ISGs) ${ }^{4}$. Proteins that are encoded by ISGs then target crucial steps in the viral life cycle and also regulate innate immune sensing and the production of cytokines, thereby establishing an antiviral state. Cytokines and chemokines that are produced following the activation of PRR signals are also crucial for shaping an effective adaptive immune response.

Viruses have evolved effective mechanisms to avoid detection by innate immune sensors, or to inhibit the activation of PRRs and/or their downstream signalling cascades. In this Review, we discuss the cell-intrinsic mechanisms that are used to sense viral pathogens in infected host cells, with a focus on RLRs, cGAS and IFI16, which are key intracellular sensors of viral RNA or viral DNA. Furthermore, we describe the strategies used by viral pathogens to evade or subvert the innate immune response that is mediated by these sensors, with a focus on recently published work and clinically important viruses. These evasion strategies fall into four broad categories: the sequestration or modification of viral nucleic acids; interference with specific post-translational modifications of PRRs or their adaptor proteins; the degradation or cleavage of PRRs or their adaptor proteins; and the sequestration or relocalization of PRRs. Finally, we detail how a comprehensive understanding of the manipulation of immune surveillance pathways by viruses may guide the design of antiviral therapeutics and vaccines, or help to improve the immunogenicity of viral vectors.

\section{Recognition of viral nucleic acids}

Intracellular sensing of viral RNA. Over the past few years, several cytoplasmic viral RNA sensors have been discovered (BOX 1) that detect the genomic RNA of invading virions, RNA replication intermediates or transcription products, and subsequently induce type I IFN responses. Furthermore, some NLR family members have been implicated in sensing viral nucleic acids, such as single-stranded RNA (ssRNA; BOXES 1,2), which trigger the induction of IFN responses or the activation of the inflammasome. Among the different RNA sensors, DExD/ $\mathrm{H}$-box RNA helicases of the RLR family have been identified as essential sensors of RNA viruses. The three RLR members, retinoic acid-inducible gene-I protein (RIG-I), melanoma differentiation-associated protein 5 (MDA5; also known as IFIH1) and LGP2 (also known as DHX58), are expressed in most host cell types and have a central helicase domain and a carboxy-terminal domain, which are both required for RNA binding ${ }^{5,6}$. In addition, RIG-I and MDA5 have a tandem caspase activation and recruitment domain (CARD) signalling module at the amino terminus, which enables them to initiate innate immune signalling following the recognition of viral RNA. By contrast, LGP2, which lacks a CARD signalling domain, negatively regulates RIG-I signalling, while promoting the binding of RNA to MDA5 (reviewed in REF. 7). RIG-I and
MDA5 detect distinct viral RNA PAMPs based on their unique features, which are not usually found in mature RNA species in host cells (BOX 2). Studies on viral infection in human and mouse cells and mice that lack RIG-I and/or MDA5 have demonstrated the importance of both sensors in the detection of viral RNA. RIG-I is crucial for the recognition of many negative-strand RNA viruses (for example, rhabdoviruses, influenza viruses and arenaviruses), as well as the recognition of Japanese encephalitis virus (JEV) and HCV, which have positive-strand RNA genomes ${ }^{3,8,9}$. By contrast, MDA5 senses the RNA of picornaviruses. Furthermore, recent studies have shown that many viruses, including paramyxoviruses, certain flaviviruses (for example, dengue virus (DENV) and West Nile virus (WNV)) and reoviruses, are sensed by both RIG-I and MDA5 (REF. 9), and it has been demonstrated that different RNA species produced during infection with these viruses are detected by RIG-I and MDA5 in a temporal-dependent manner (reviewed in REFS 3,10). In addition, there is accumulating evidence that RIG-I has a role in the detection of several DNA viruses (for example, Epstein-Barr virus (EBV), Kaposi sarcoma-associated herpesvirus (KSHV), herpes simplex virus 1 (HSV-1) and adenoviruses $)^{3,11,12}$, in some cases by recognizing RNA species that are generated by RNA polymerase III (BOX 1).

The signal-transducing activity of RIG-I and MDA5 is controlled by post-translational modifications and regulatory host enzymes (reviewed in REF. 13). In uninfected cells, RIG-I and MDA5 are constitutively phosphorylated at specific serine or threonine residues in their CARDs and C-terminal domains, which keeps them in a signallingrepressed state ${ }^{14-18}$ (FIG. 1). In addition, RIG-I is held in an auto-repressed conformation through an intramolecular interaction between the helicase domain and the CARDs $^{19}$, whereas MDA5 may exhibit an open conformation in the absence of foreign RNA. Following the binding of RNA, RIG-I and MDA5 undergo a conformational change ${ }^{20}$ that is dependent on their ATPase activity, which is stimulated by the binding of protein kinase $\mathrm{R}(\mathrm{PKR})$ activator (PACT ${ }^{21}$. This conformational change releases the CARDs, which bind to several regulatory molecules. Both RIG-I and MDA5 recruit the phosphatase PP1 - PP1 1 or PP1 $\gamma$ isoform - which removes the inhibitory phosphorylation marks in their CARDs $^{16}$. In the case of RIG-I, the E3 ubiquitin ligases tripartite motif protein 25 (TRIM25) and Riplet (also known as RNF135) are subsequently recruited and attach Lys63-linked ubiquitin polymers onto the CARDs and C-terminal domain, respectively ${ }^{22,23}$. Lys63-linked ubiquitylation is crucial for the tetramerization of RIG-I and its interaction with the adaptor protein MAVS at the outer membrane of mitochondria and mitochondria-associated membranes (MAMs) ${ }^{22,24,25}$. Conversely, the removal of Lys63-linked ubiquitylation by the cellular deubiquitylating enzymes (DUBs) ubiquitin C-terminal hydrolase 3 (USP3), USP21 and CYLD, represses RIG-I signalling (reviewed in REF. 13). Recently, it has been shown that 14-3-3e, a mitochondrial trafficking protein, interacts with RNA-bound RIG-I in complex with TRIM25, which results in the translocation of activated RIG-I to mitochondria for binding to MAVS ${ }^{26}$. Whether a 


\section{Box 1 | Other intracellular sensors of viral infection}

Mitochondrial antiviral signalling protein

(MAVS).A transmembrane adaptor protein that is found primarily on mitochondria, but also on peroxisomes. The activation of MAVS by RIG--like receptors (RLRS) leads to the formation of large MAVS aggregates that drive the activation of interferon regulatory factor 3 (IRF3) and the subsequent expression of interferon genes to establish an antiviral state.

\section{Inflammasome}

A large multiprotein complex that activates caspase 1 , which leads to the release of mature forms of the pro-inflammatory cytokines interleukin- $1 \beta$ (IL-1 $\beta$ ) and $\mathrm{IL}-18$ and results in pro-inflammatory cell death Activation of the inflammasome can be triggered by pathogen-associated molecular patterns (PAMPs) or danger signals

DExD/H-box RNA helicases A large family of proteins that uses the energy from ATP hydrolysis to unwind RNA molecules or dissociate RNA-protein complexes for the modulation of RNA structures. These proteins have important roles in RNA metabolism transcriptional regulation and the innate immune sensing of foreign RNA.

Tripartite motif protein 25 (TRIM25). A member of a family of approximately 80 proteins in humans that is characterized by the "tripartite motif', which is comprised of a RING domain, one or two B-boxes and a coiled-coil domain. In addition to their roles in fundamental cellular processes, TRIM proteins directly block crucial steps in the viral life cycle, or they modulate pattern recognition receptor (PRR) signalling.

Deubiquitylating enzymes (DUBs). Proteases that cleave the peptide or isopeptide bond between ubiquitin and its target protein. The human genome encodes approximately 100 DUB enzymes; in addition, viruses can encode DUBs to manipulate ubiquitin-dependen processes in the infected host cell.

\section{Viral RNA sensors}

PKR. Protein kinase R (PKR) is known to sense viral double-stranded RNA (dsRNA), inhibit cap-dependent translation by eukaryotic initiation factor $2 \alpha$ (elF2 $\alpha$ ) and activate inflammasomes and autophagy (reviewed in REF. 132). Recently, PKR was shown to induce the formation of stress granules, which may function as platforms to facilitate the interaction of RIG-I-like receptors (RLRs) and viral RNA (reviewed in REF. 133).

OAS. Following the binding of viral dsRNA, oligoadenylate synthase (OAS) produces 2'-5'-linked oligoadenylate, activating RNase L, which degrades cellular and viral RNA to block virus replication (reviewed in REF. 134). RNA fragments that are generated by RNase $\mathrm{L}$ can also act as retinoic acid-inducible gene-I protein (RIG-I) ligands, which potentiate the production of interferon (IFN) ${ }^{135}$.

Non-RLR DExD/H helicases. Several DExD/H helicases besides RLRs have been proposed to function as viral RNA sensors; however, these proteins are also implicated in host RNA metabolism or have immunoregulatory roles. For example, DDX3 (also known as DDX3X) was proposed to function as a viral RNA sensor, inducing the production of IFN $\beta$ through mitochondrial antiviral signalling protein (MAVS); however, DDX3 was also shown to regulate the activity of TBK1 and $I_{\kappa} B$ kinase- $\varepsilon(\mathrm{IKK \varepsilon})$, and to promote PKR activator (PACT) mRNA translation (reviewed in REF. 3). DDX60 acts as a cofactor for the activation of RIG-I and is also involved in the degradation of viral RNA ${ }^{136}$.

\section{Viral DNA sensors}

DAI. DNA-dependent activator of IFN-regulatory factors (DAI) was the first cytosolic viral DNA sensor to be discovered, which responds to poly(deoxyadenylic-deoxythymidylic) (poly(dA:dT)) DNA. Following DNA detection, DAl induces the production of type I IFNs through IFN regulatory factor 3 (IRF3) and nuclear factor- $\mathrm{B}$ (NF- $\kappa B$ ). However, mice and some human cells that are deficient in DAI have an intact immune response to viral DNA, which suggests a redundant or cell type-specific role for DAI (reviewed in REF. 33).

RNA polymerase III. Besides its known role in the synthesis of small cellular RNAs, RNA polymerase III (Pol III) has been shown to convert AT-rich viral double-stranded DNA (dsDNA) into small RNA that has a $5^{\prime}$-triphosphate group that subsequently activates RIG-I (reviewed in REF. 33).

AIM2. Absent in melanoma 2 (AIM2), a member of the pyrin and HIN200 domain-containing protein (PYHIN) family, has also been implicated in viral DNA sensing. AIM2 triggers the caspase-1-dependent activation of the inflammasome, which leads to the production of interleukin-1 $\beta$ (IL-1 $\beta$ ) and IL-18 (REF. 137). Host cells that are deficient in AIM2 have defective innate immune responses to infection with certain DNA viruses, such as vaccinia virus and mouse cytomegalovirus ${ }^{138}$.

Other DNA sensors. Several other putative DNA sensors have been described, which include DDX41, DHX9, DHX36, DNA-dependent protein kinase (DNA-PK) and MRE11 (reviewed in REF. 33). Further work is required to establish which proteins are bona fide sensors of DNA, or whether they have cell type-specific roles.

\section{NLRs}

NOD-like receptors (NLRs) detect diverse intracellular pathogen-associated molecular patterns (PAMPs) or danger signals that stem from microbial infection. Among the 22 NLRs that are encoded in the human genome, nucleotide-binding oligomerization domain-containing protein 2 (NOD2) and NOD-, LRR- and pyrin domain-containing 3 (NLRP3) have the most well-characterized roles with regard to viral infection and viral nucleic acid sensing ${ }^{139,140}$. NOD2 has been shown to induce the production of IFNs following transfection of single-stranded RNA (ssRNA) or infection with respiratory syncytial virus (RSV) and influenza A virus (IAV), whereas the activation of the NLRP3 inflammasome is triggered by infection with IAV and encephalomyocarditis virus (EMCV) ${ }^{141-144}$. However, it is unclear whether NLRP3 directly senses viral RNA.

Lys63-polyubiquitin mark is necessary for the multimerization and signalling of MDA5 is still a subject of debate, as the binding of MDA5 to long double-stranded RNA (dsRNA) is sufficient to induce the formation of MDA5 filaments and binding to MAVS ${ }^{27}$. In addition to mitochondria and/or MAMs, MAVS is found at peroxisomes where it initiates downstream signalling that preferentially induces the expression of type III IFN genes ${ }^{28}$. Once activated, MAVS multimerizes into prion-like filament structures ${ }^{29}$, which nucleate the assembly of a large 'signalosome' complex that is composed of the tumour necrosis factor (TNF) receptor-associated factor (TRAF) proteins,

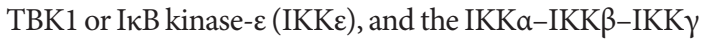
ternary complex, which leads to the activation of IFN regulatory factor 3 (IRF3) and/or IRF7 and nuclear factor- $\mathrm{kB}$ $(\mathrm{NF}-\kappa \mathrm{B})$. IRF3, IRF7 and NF- $\kappa \mathrm{B}$ together with activator protein 1 (AP1) then induce the transcriptional activation of IFNs, other cytokines (for example, TNF, interleukin-6
(IL-6) and IL-8), and numerous ISGs to create an antiviral milieu in infected and uninfected neighbouring host cells. Intriguingly, it has been recently reported that RIG-I and MDA5 can also block viral replication independent of the induction of cytokine gene expression by inhibiting the interaction of viral proteins with viral $\mathrm{RNA}^{30-32}$.

Intracellular sensing of viral DNA. It has long been recognized that the introduction of foreign DNA into the cytoplasm of mammalian cells induces an innate immune response. Over the past few years, several intracellular DNA sensor candidates have been identified (BOX 1 and reviewed in greater detail in REF. 33) as well as an essential adaptor protein, STING, through which most of these sensors operate ${ }^{34-36}$. Although the functional relevance of some of these DNA sensors still needs to be fully established, cGAS and IFI16 have been identified as bona fide intracellular viral DNA receptors. 


\section{Box 2 | Viral PAMPs and their structural features}

\section{RLR ligands}

Both retinoic acid-inducible gene-I protein (RIG-I) and melanoma differentiation-associated protein 5 (MDA5) can recognize the synthetic double-stranded RNA (dsRNA) analogue polyinosinic-polycytidylic acid (poly(l:C)), but RIG-I preferentially senses short dsRNA, whereas MDA5 responds to high molecular weight (HMW)-poly(l:C $)^{3,8,10}$. Importantly, a $5^{\prime}$-triphosphate or $5^{\prime}$-diphosphate moiety on the RNA (5'-ppp-RNA or 5'-pp-RNA), which is not found in most mature cellular RNAs, is essential for recognition by RIG-I, but not MDA5 (REFS 145-147). In addition to the $5^{\prime}$-triphosphate or 5'-diphosphate group, recognition by RIG-I requires adjacent base-paired structures, such as hairpin or panhandle conformations, as found in the genomes of many RNA viruses, including influenza A virus (IAV). Specific sequences, such as poly-U or poly-UC motifs that are found in hepatitis $C$ virus (HCV) RNA, also contribute to efficient recognition by RIG-I 148,149 . Research on physiological pathogen-associated molecular patterns (PAMPs) during an authentic viral infection has shown that RIG-I recognizes genomic RNAs as well as the RNA of defective interfering particles of Sendai virus and IAV ${ }^{150,151}$. RIG-I also senses the $5^{\prime}$ - $\varepsilon$-region of the hepatitis $B$ virus (HBV) pre-genomic $R N A^{30}$.

Apart from HMW-poly(I:C), MDA5 has been shown to be stimulated by large web-like RNA aggregates that are produced during infection with encephalomyocarditis virus (EMCV); these aggregates contain both single-stranded RNA (ssRNA) and dsRNA regions ${ }^{152}$. In addition, ribose $2^{\prime}$-O-methylation of the $5^{\prime}$-cap structure is required to avoid the activation of MDA5 (REF. 153).

\section{cGAS and IFI16 ligands}

Cyclic GMP-AMP synthase (cGAS) senses the double-stranded DNA (dsDNA) of many DNA viruses by directly binding to the sugar-phosphate backbone, which indicates the sequence-independent sensing of viral DNA (reviewed in REF. 134). cGAS also detects HIV-1 and other retroviruses by sensing reverse transcribed viral DNA ${ }^{38,110,111}$. Intriguingly, HIV-1 'strong-stop' DNA (sstDNA) activates cGAS in a sequence-dependent manner ${ }^{154}$. In contrast to cGAS, interferon- $\gamma$ (IFN $\gamma$ )-inducible protein 16 (IFI16) is predominantly located in the nucleus where it senses the DNA of herpes simplex virus 1 (HSV-1) and Kaposi sarcoma-associated herpesvirus (KSHV) ${ }^{121}$. Other studies have shown that IFI16 also binds to cytosolic viral DNA, such as HIV-1 single-stranded DNA (ssDNA) that has secondary structures (reviewed in REFS 49, 155). cGAS and IFI16 may cooperate to sense viral DNA and initiate innate immune signalling ${ }^{156}$.

\section{Viral PAMPs detected by NLRs}

Nucleotide-binding oligomerization domain-containing protein 2 (NOD2) detects viral ssRNA, whereas NOD-, LRR- and pyrin domain-containing 3 (NLRP3) may sense several PAMPs, including viral RNA or DNA in combination with ATP, ion flux or reactive oxygen species ${ }^{141-143,157,158}$. NOD2 associates with the adaptor mitochondrial antiviral signalling protein (MAVS) for the production of type I IFNs, whereas activated NLRP3 inflammasomes signal through the adaptor protein apoptosis-associated speck-like protein containing a CARD (ASC) to trigger the activation of caspase 1 and the production of interleukin-1 $\beta$ (IL-1 $\beta$ ) and IL-18.

In the cytoplasm, cGAS binds to viral double-stranded DNA (dsDNA) that is derived from various sources, including DNA viruses and retroviruses, of which the latter generate DNA through the reverse transcription of their RNA genomes ${ }^{37-40}$ (BOX 2; FIG. 2). Following the binding of DNA, cGAS produces the cyclic dinucleotide cyclic GMP-AMP (cGAMP) $)^{41,42}$, which is characterized by an unusual $2^{\prime}-5^{\prime}$ phosphodiester bond similar to $2^{\prime}-5^{\prime}$ oligoadenylate, which is generated by oligoadenylate synthase (OAS) ${ }^{43}$. The second messenger cGAMP subsequently binds to STING in the same host cell, which leads to its activation. In addition, cGAMP can spread from infected host cells to neighbouring cells through gap junctions, mediating the activation of STING in bystander cells to impede viral infection ${ }^{44}$. Following the binding of cGAMP, STING dimerizes and undergoes Lys63-linked ubiquitylation mediated by TRIM32 and TRIM56 (REFS 45,46); these two steps are crucial for the activation of STING. Once activated, STING then translocates from the endoplasmic reticulum to the Golgi apparatus and perinuclear structures where it interacts with TBK1 and is subsequently phosphorylated ${ }^{47}$, which triggers the activation of IRF3 (REF. 48). Furthermore, STING promotes the activation of NF- $\mathrm{\kappa B}$.

Another key cytoplasmic sensor of viral dsDNA is IFI16, which is a member of the pyrin and HIN200 domain-containing protein family (PYHIN protein family) ${ }^{49}$.
Following the binding of viral dsDNA (BOX 2; FIG. 2), IFI16 assembles into filaments and binds to STING, thereby triggering the IRF3-dependent and NF- $\mathrm{BB}$-dependent expression of IFN genes. Furthermore, IFI16 activates the inflammasome, which results in the generation of active caspase 1 and the production of IL-1 $\beta^{50}$. Intriguingly, evidence has emerged that in many cell types IFI16 also localizes to the nucleus, which indicates that IFI16 can distinguish between 'self' and 'non-self' DNA in the nucleus, the site of replication of most DNA viruses - for example, herpesviruses, papillomaviruses and polyomaviruses. However, future studies are required to determine the molecular details of how IFI16 recognizes viral DNA in the nucleus, and how it signals to activate the STING-TBK1-IRF3 axis in the cytoplasm.

\section{Evasion of RLR-MAVS-dependent immunity}

All successful viral pathogens have effective strategies to evade or inhibit the activation of intracellular PRRs. In this section, we discuss the molecular strategies that are used by viruses to inhibit the activation of RLRs and MAVS. Many viruses also inhibit innate immune responses by targeting downstream molecules that are shared between RLRs and other PRRs, such as TBK1, IRF3, IRF7 and NF- $\kappa$ B, or they block $\mathrm{IFNa} / \beta$ receptor signalling or the function of specific 


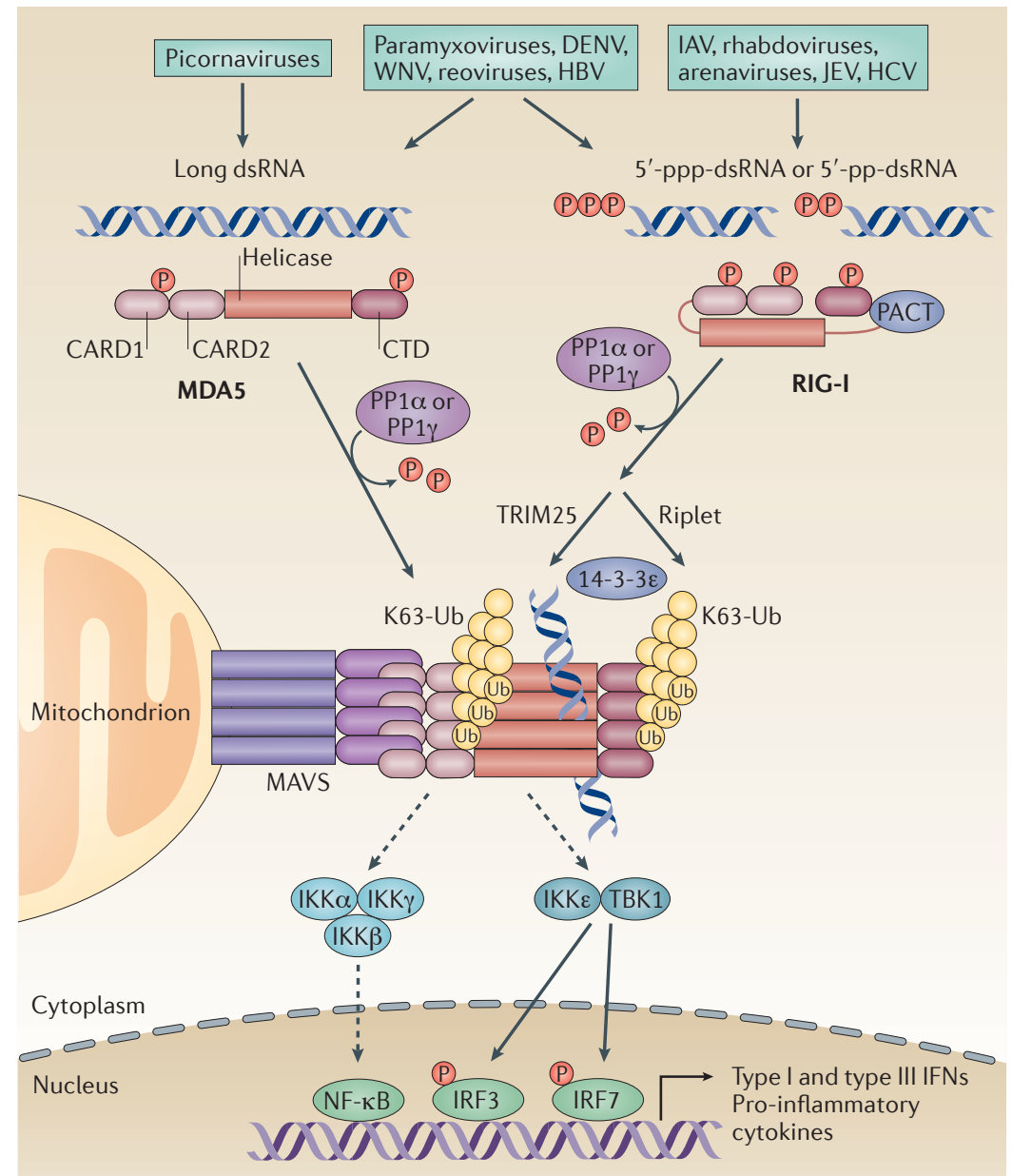

Figure 1 | RLR-MAVS-mediated signal transduction pathway. RIG-I-like receptors (RLRs) have been identified as important cytoplasmic viral RNA sensors that recognize the genomic RNA and/or RNA replication intermediates of numerous viruses. The two RLR members retinoic acid-inducible gene-I protein (RIG-I) and melanoma differentiation-associated protein 5 (MDA5) are kept inactive in uninfected cells through the phosphorylation of their caspase activation and recruitment domains (CARDs) and carboxy-terminal domains (CTDs). In addition, RIG-I adopts a 'closed' auto-inhibited conformation. Following viral infection, RIG-I recognizes cytoplasmic viral short double-stranded RNA (dsRNA) that contains a 5'-triphosphate or 5'-diphosphate moiety, whereas MDA5 detects long dsRNA structures. Following the binding of RNA, RIG-I and MDA5 are dephosphorylated by PP1 $\alpha$ or PP1 $\gamma$, which induces the formation of a signalling-active CARD conformation. RIG-I is further activated by the Lys63-linked ubiquitylation of its CARDs that is mediated by tripartite motif protein 25 (TRIM25). Riplet, another E3 ubiquitin ligase, mediates the Lys63-linked ubiquitylation of the CTD of RIG-I, which is also crucial for the activation of RIG-I. Lys63-linked ubiquitylation induces the tetramerization of RIG-I (the signalling-active form of RIG-I), which subsequently interacts with the adaptor mitochondrial antiviral signalling protein (MAVS) on mitochondria, mitochondria-associated membranes (MAMs) or peroxisomes (not shown). The mitochondrial-targeting chaperone protein $14-3-3 \varepsilon$ is essential for the translocation of RIG-I to mitochondrial MAVS. In the case of MDA5, binding to long dsRNA induces MDA5 filament formation, which subsequently enables MDA5 to bind to MAVS. MAVS activates TBK1 or I $\mathrm{KB}$ kinase- $\varepsilon(\mathrm{IKK} \varepsilon$ ) as well as the IKKa-IKK $\beta-I K K \gamma$ complex, which activate interferon (IFN) regulatory factor 3 (IRF3) and IRF7, and nuclear factor- $\kappa \mathrm{B}(\mathrm{NF}-\kappa \mathrm{B})$, respectively, through phosphorylation events. IRF3 and/or IRF7 and $\mathrm{NF}-\kappa \mathrm{B}$ together with activator protein 1 (AP1; not illustrated) induce the gene expression of type I IFNs (mainly IFNa subtypes and IFN $\beta$ ), type III IFNs (IFN $\lambda$ subtypes), and many other pro-inflammatory cytokines and chemokines, to establish an antiviral state. Solid arrows indicate direct signalling events. Dashed arrows indicate indirect signalling events. DENV, dengue virus; $\mathrm{HBV}$, hepatitis $B$ virus; $H C V$, hepatitis $C$ virus; IAV, influenza $A$ virus; JEV, Japanese encephalitis virus; K63-Ub, Lys63-linked ubiquitylation; P, phosphate; PACT, protein kinase R activator; Ub, ubiquitin; WNV, West Nile virus. antiviral effector proteins. However, these strategies are beyond the scope of this Review and have been discussed extensively elsewhere ${ }^{51,52}$.

Sequestration or modification of viral RNA ligands. Most RNA viruses replicate in the cytoplasm where RLRs are also present and well positioned to detect foreign RNA. Several viruses have evolved ways to sequester their genomes to escape surveillance by RLRs (FIG. 3). A major strategy that is used by viruses to prevent RLRs from accessing viral RNA is to induce the formation of specific replication compartments that are confined by cellular membranes, or to replicate on organelles, such as the endoplasmic reticulum, the Golgi apparatus and mitochondria. For example, DENV, a mosquito-borne flavivirus that causes dengue fever and the more severe dengue haemorrhagic fever, replicates in convoluted membranes of the endoplasmic reticulum, which efficiently conceal dsRNA from the cytosol, thus preventing the activation of RLRs. By contrast, JEV, a related flavivirus, fails to conceal dsRNA and markedly induces the production of type I IFNs ${ }^{53}$. Unlike members of the Flaviviridae family and most other RNA viruses, influenza A viruses (IAVs), which are responsible for seasonal outbreaks of flu, have atypical life cycles and replicate in the nucleus to avoid the sensing of viral RNA by RLRs in the cytoplasm. In fact, IAV contains eight RNA genome segments that would potently stimulate the activation of RIG-I if found in large quantities in the cytoplasm. In addition, IAV has developed a strategy to avoid the recognition of viral RNA during the short transit of the virus through the cytoplasm after viral entry. It has been shown that in some strains of IAV the viral polymerase subunit PB2 prevents the RIG-I-mediated recognition of incoming genomic RNAs, which are encapsidated by nucleoproteins (NPs) ${ }^{31}$. PB2 in mammalian-adapted strains of IAV harbours a lysine residue at position 627 and has an increased affinity for NP, and this tight packing of the viral genome hinders the binding of RIG-I. Similarly, other viruses use viral or host-encoded proteins that 'shield' viral RNA from RLRs. For example, viral protein 35 (VP35) from EBOV and Marburg virus, non-structural protein 1 (NS1) from IAV and the E3 protein from vaccinia virus bind to viral dsRNA to avoid detection by RIG-I ${ }^{54-59}$, whereas respiratory syncytial virus (RSV), a member of the Paramyxoviridae family that can cause severe infection of the respiratory tract especially in children, uses the host cellular RNA-binding protein La to prevent RIG-I from binding to viral leader RNA ${ }^{60}$. Finally, the $\mathrm{C}$ protein of human parainfluenza virus type 1 (HPIV1) has been shown to limit the accumulation of cytoplasmic dsRNA to prevent the activation of MDA5 and the expression of type I IFN genes ${ }^{61}$.

As RLRs specifically recognize certain features of viral nucleic acids (BOX 2), several viruses modify their genomes to prevent detection or hinder the activation of RLRs. Members of the Bunyaviridae family, such as Hantaan virus, and Crimean-Congo haemorrhagic fever virus (CCHFV), and also Borna disease virus (BDV) in the Bornaviridae family, encode phosphatases 


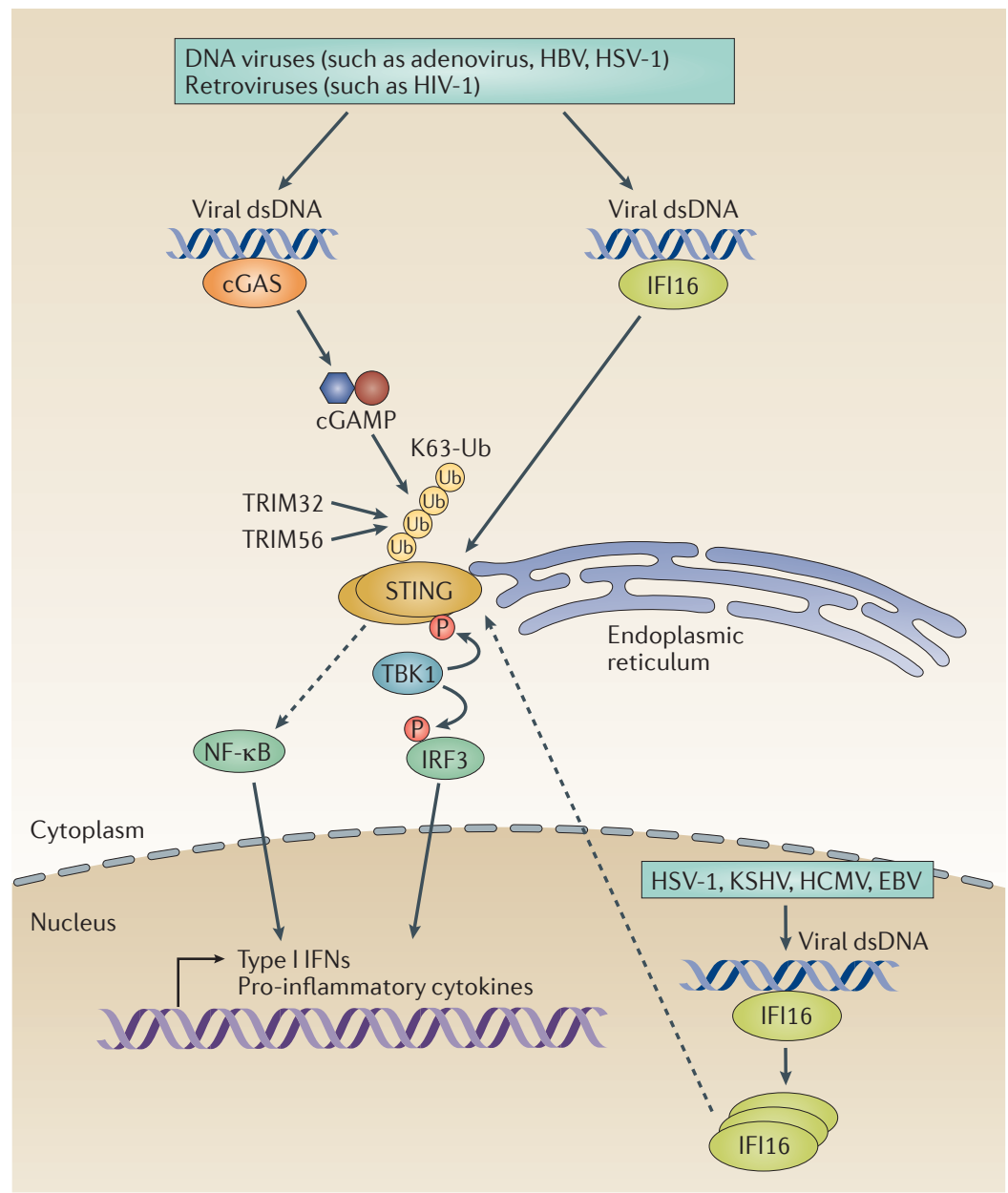

Figure 2 | Antiviral signalling mediated by cGAS and IFI16 through STING. In the cytoplasm of infected cells, cyclic GMP-AMP synthase (cGAS) recognizes double-stranded DNA (dsDNA) from DNA viruses or dsDNA that is produced by retroviruses through the reverse transcription of their RNA genomes. Following the binding of DNA, cGAS synthesizes the second messenger cyclic GMP-AMP (cGAMP), which then binds to and activates stimulator of interferon (IFN) genes (STING) on the endoplasmic reticulum. STING is further activated by dimerization and Lys63-linked ubiquitylation that is mediated by tripartite motif protein 32 (TRIM32) and TRIM56. Furthermore, STING is phosphorylated by TBK1. The sensor IFN $\gamma$-inducible protein 16 (IFI16) senses viral dsDNA in both the cytoplasm and the nucleus. Following the binding of viral DNA, IFI16 multimerizes and then signals through STING in the cytoplasm. The activation of STING induces the expression of type I IFN genes and other pro-inflammatory cytokines through the TBK1-IFN regulatory factor 3 (IRF3) axis and nuclear factor- $\kappa \mathrm{B}(\mathrm{NF}-\kappa \mathrm{B})$. Solid arrows indicate well-established signalling events. Dashed arrows indicate signalling events that are indirect or that have not yet been fully elucidated. EBV, Epstein-Barr virus; HBV, hepatitis B virus; HCMV, human cytomegalovirus; HSV-1, herpes simplex virus 1; K63-Ub, Lys63-linked ubiquitylation; KSHV, Kaposi sarcoma-associated herpesvirus; P, phosphate; Ub, ubiquitin.

to process the $5^{\prime}$-triphosphate group on their genomes to $5^{\prime}$-monophosphate to escape surveillance by RIG- $\mathrm{I}^{62,63}$. Arenaviruses, such as Junin virus, which can cause haemorrhagic fever in humans, have a $5^{\prime}$-triphosphate group on their genomes, but with an atypical $5^{\prime}$ unpaired nucleotide overhang, which does not trigger the production of type I IFNs ${ }^{64}$. Mechanistically, $5^{\prime}$-triphosphate dsRNA with a nucleotide overhang binds to RIG-I without causing its activation, thereby acting as a decoy $\mathrm{RNA}^{65}$. Lassa virus has evolved another unique strategy in which the C-terminal half of its nucleoprotein (NP) adopts a 3D fold similar to the DEDD superfamily of exonucleases and has authentic $3^{\prime}-5^{\prime}$ exonuclease activity. This activity enables the NP from Lassa virus to digest free dsRNA, which prevents the activation of RIG-I ${ }^{66,67}$.

Manipulation of the post-translational modification of RLRs and MAVS. In recent years, it has become clear that the RLR signalling pathway is intricately regulated by post-translational modifications - ubiquitylation and serine/threonine phosphorylation in particular of RLRs and downstream signalling molecules. As the Lys63-linked ubiquitylation of RIG-I is a crucial step for its activation, it is not surprising that several viruses target this type of ubiquitylation to inhibit RIG-I signalling (FIG. 3). Some viruses directly target the cellular E3 ubiquitin ligases that are responsible for the ubiquitylation of RIG-I. For example, the NS1 proteins of many strains of IAV interact directly with TRIM25 through its coiledcoil domain; this inhibits the homo-oligomerization of TRIM25, which is crucial for its enzymatic activity to attach Lys63-linked polyubiquitin to Lys172 in the CARDs of RIG-I ${ }^{68}$. The NS1 proteins of some IAV strains can also bind to Riplet, which inhibits the Lys63-linked polyubiquitylation of RIG-I at its C-terminal domain ${ }^{69}$. Similarly, the NS3-NS4A protease complex of HCV cleaves Riplet to prevent the Lys63-ubiquitylation of RIG-I ${ }^{23}$. Other viruses - both RNA viruses and DNA viruses - directly remove the Lys63-linked ubiquitylation of RIG-I through virus-encoded DUBs, such as ORF64 from KSHV, papain-like protease (PLP) from severe acute respiratory syndrome (SARS)-associated coronavirus (SARS-CoV), leader proteinase $\left(\mathrm{L}^{\mathrm{pro}}\right)$ from foot-and-mouth disease virus (FMDV) and the ovarian tumour (OTU)-type DUBs of arteriviruses and nairoviruses ${ }^{70-73}$. Furthermore, viruses can inhibit the Lys63-linked ubiquitylation of RIG-I independent of protein-protein interactions, by modulating the abundance of cellular microRNAs or through RNA-protein interactions. For example, the $3 \mathrm{C}$ protein of enterovirus 71 (EV71), a member of the Picornaviridae family that causes hand, foot and mouth disease, and occasionally severe central nervous system diseases, downregulates the host microRNA miR-526a to increase the expression of the cellular DUB enzyme CYLD, thus inhibiting the activation of RIG-I ${ }^{74}$. More recently, the subgenomic flavivirus RNA (sfRNA) of an epidemic strain of DENV that was isolated in Puerto Rico has been shown to bind to TRIM25 in a sequence-specific manner and prevent its deubiquitylation ${ }^{75}$, which has been shown to be crucial for sustained RIG-I signalling ${ }^{76}$.

The phosphorylation of serine or threonine residues keeps RIG-I and MDA5 inactive in the absence of an infection, whereas the recruitment of PP1 $1 \alpha$ or PP1 $\gamma$ and the dephosphorylation of specific phosphorylation marks in RLRs are crucial for innate immune activation following viral infection. Measles virus $(\mathrm{MeV})$, a morbillivirus in the Paramyxoviridae family, uses its non-structural $\mathrm{V}$ protein to bind to and sequester PP1 $\alpha$ and $\mathrm{PP} 1 \gamma$ from MDA5, a mechanism that relies on the presence 


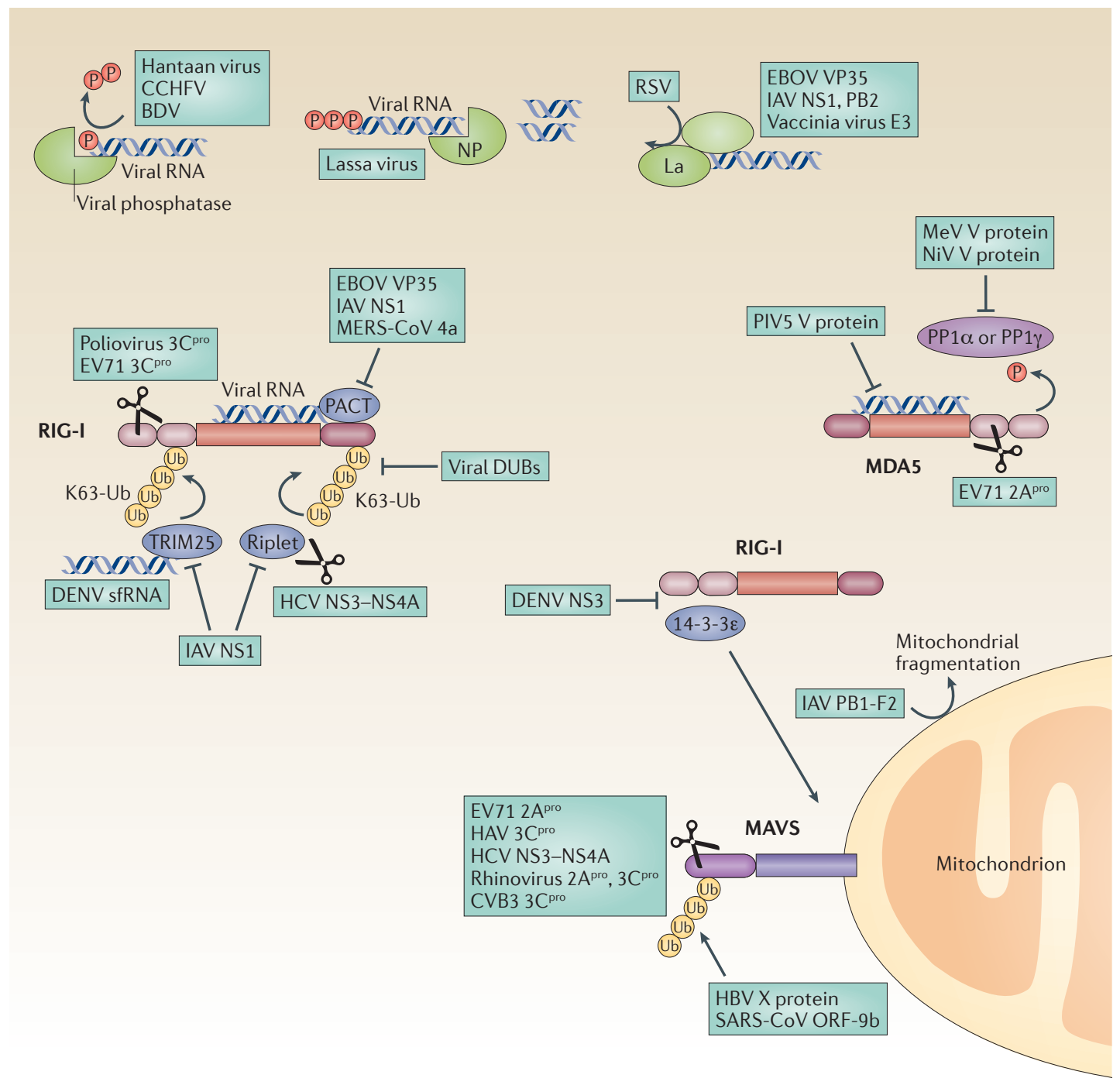

Figure 3 | Viral immune evasion of RLR-MAVS signalling. Most successful viral pathogens are equipped with effective strategies to evade or inhibit the activation of intracellular pattern recognition receptors (PRRs), such as retinoic acid-inducible gene-I protein (RIG-I) or melanoma differentiation-associated protein 5 (MDA5), or the activation of their adaptor mitochondrial antiviral signalling protein (MAVS). To prevent the activation of RIG-I, viral phosphatases can process the $5^{\prime}$-triphosphate moiety in the viral RNA, or viral nucleases, such as the nucleoprotein (NP) of Lassa virus, can digest free double-stranded RNA (dsRNA). Furthermore, viral proteins, such as viral protein 35 (VP35) from EBOV, non-structural protein 1 (NS1) or PB2 from influenza A virus (IAV) and the E3 protein from vaccinia virus, or host proteins (such as La) bind to viral RNA to inhibit the recognition of pathogen-associated molecular patterns (PAMPs) by RIG-I. Several viruses manipulate specific post-translational modifications of RIG-I and/or MDA5, thereby blocking their signalling abilities. For example, viruses prevent the Lys63-linked ubiquitylation of RIG-I by encoding viral deubiquitylating enzymes (DUBs). NS1 from IAV and the NS3-NS4A protease complex from hepatitis C virus (HCV) antagonize the cellular E3 ubiquitin ligases, tripartite motif protein 25 (TRIM25) and/or Riplet, thereby also inhibiting RIG-I ubiquitylation and thus its activation. Furthermore, subgenomic flavivirus RNA (sfRNA) from dengue virus (DENV) binds to TRIM25 to block sustained RIG-I signalling. To suppress the activation of MDA5, the $V$ proteins from measles virus (MeV) and Nipah virus (NiV) prevent the PP1 $\alpha$-mediated or PP1 $\gamma$-mediated dephosphorylation of MDA5, keeping it in its phosphorylated inactivate state, whereas the $V$ protein of parainfluenza virus 5 (PIV5) blocks the ATPase activity of MDA5. Furthermore, VP35 from EBOV, NS1 from IAV and the 4a protein from Middle East respiratory syndrome coronavirus (MERS-CoV) target protein kinase R activator (PACT) to antagonize RIG-I. The NS3 protein from DENV targets the trafficking factor $14-3-3 \varepsilon$ to prevent the translocation of RIG-I to MAVS at the mitochondria. Numerous viruses encode proteases (Pro) to cleave RIG-I, MDA5 and/or MAVS. PB1-F2 from IAV translocates into the mitochondrial inner membrane space to accelerate mitochondrial fragmentation. Other viruses subvert cellular degradation pathways to inhibit RLR-MAVS-dependent signalling. Specifically, the X protein from hepatitis B virus (HBV) and the $9 \mathrm{~b}$ protein from severe acute respiratory syndrome (SARS)-associated coronavirus (SARS-CoV) promote the ubiquitylation and degradation of MAVS. BDV, Borna disease virus; CCHFV, Crimean-Congo haemorrhagic fever virus; CVB3, coxsackievirus B3; EV71, enterovirus 71; HAV, hepatitis A virus; K63-Ub, Lys63-linked ubiquitylation; P, phosphate; $\mathrm{RSV}$, respiratory syncytial virus; Ub, ubiquitin. 
of a specific PP1-binding motif in the C-terminal 'tail' region of the viral protein ${ }^{77}$. Nipah virus $(\mathrm{NiV})$, a related virus that has been observed to cause encephalitis and respiratory illness during outbreaks in South Asia and Southeast Asia, is also able to target PP1 $\alpha$ and PP1 $\gamma$ and it is likely that other paramyxoviruses may have evolved a similar PP1-antagonistic strategy. The $\mathrm{V}$ proteins of $\mathrm{MeV}$ and $\mathrm{NiV}$ are, in turn, dephosphorylated by PP1 $\alpha$ or PP1 $\gamma$, which suggests that these viral proteins may act as decoy substrates; alternatively, it is possible that the dephosphorylation of the V proteins by PP1a or $\mathrm{PP} 1 \gamma$ may promote other functions of the $\mathrm{V}$ proteins that could be beneficial for viral replication and/or virusinduced pathogenesis. In addition, $\mathrm{MeV}$ has evolved a $\mathrm{V}$ protein-independent mechanism to inhibit the dephosphorylation of both RIG-I and MDA5 by PP1 $\alpha$ or PP1 $\gamma$ in dendritic cells. Specifically, $\mathrm{MeV}$ binds to the CLR protein dendritic-cell-specific ICAM3-grabbing non-integrin (DC-SIGN; also known as CD209), and triggers its activation. DC-SIGN signalling then activates the kinase RAF1, which negatively regulates the activity of PP1 $\alpha$ and PP1 $\gamma$ and inhibits the dephosphorylation of both RIG-I and MDA5 (REF. 78).

Cleavage or degradation of RLRs and MAVS. One of the most effective ways to inhibit PRR signalling is to eliminate the sensor or a key component of its signalling pathway. As such, many viruses encode viral proteases that directly cleave RLRs (FIG. 3). The $3 \mathrm{C}^{\text {pro }}$ proteases of both poliovirus and EV71 cleave RIG-I, whereas the $2 \mathrm{~A}^{\text {pro }}$ protease of EV71 cleaves MDA5 (REFS 79,80). As MAVS is crucial for both RIG-Imediated and MDA5-mediated signalling, it is not surprising that numerous viral proteases target and cleave MAVS, such as $3 \mathrm{C}^{\text {pro }}$ from hepatitis A virus (HAV), 2A $\mathrm{A}^{\text {pro }}$ from EV71, NS3-NS4A from $\mathrm{HCV}$ and $\mathrm{GB}$ virus $\mathrm{B}, 2 \mathrm{~A}^{\text {pro }}$ and $3 \mathrm{C}^{\text {pro }}$ from rhinovirus, and $3 \mathrm{C}^{\text {pro }}$ from coxsackievirus B3 (CVB3) $)^{80-85}$. By contrast, IAV uses a non-proteolytic mechanism to degrade MAVS. Specifically, PB1-F2 from IAV, a small accessory protein that contributes to viral pathogenicity, translocates into the mitochondrial inner membrane space to reduce the inner membrane potential, which accelerates the fragmentation of mitochondria and thereby inhibits MAVS signalling $^{86}$. Furthermore, PB1-F2 binds to the transmembrane region of MAVS to block the induction of IFN production ${ }^{87}$.

Another strategy that is used by viruses to decrease the abundance of RLRs and MAVS is to subvert cellular degradation pathways. The X protein of hepatitis B virus (HBV), a DNA virus in the Hepadnaviridae family that also produces RNA species during its life cycle, binds to MAVS and promotes its degradation through the ubiquitylation of Lys136; however, the identity of the cellular ubiquitylating enzyme that is involved is unknown $^{88}$. Infection with poliovirus leads to MDA5 being cleaved independent of the viral proteases $2 \mathrm{~A}^{\text {pro }}$ and $3 C^{\text {pro }}$. Instead, the cleavage of MDA 5 occurs in a proteasome-dependent and caspase-dependent manner ${ }^{89}$. Moreover, infection with $\mathrm{MeV}$ triggers selective autophagy to degrade mitochondria, a process termed mitophagy, which decreases the abundance of MAVS ${ }^{90}$. SARS-CoV, which causes severe acute respiratory syndrome, has evolved a strategy in which its $9 \mathrm{~b}$ protein localizes to mitochondria and subverts the cellular E3 ubiquitin ligase atrophin-1-interacting protein 4 (AIP4) to degrade MAVS ${ }^{91}$. Another study showed that the NS1 and NS2 proteins of RSV trigger the proteasomedependent degradation of RIG-I and numerous other immune molecules, but not MAVS, by assembling a large degradative complex on mitochondria ${ }^{92}$. More recently, a novel mechanism has been proposed for DENV and WNV in which their capsid proteins bind to peroxisomal biogenesis factor 19 (PEX19), which leads to a decrease in the number of peroxisomes in the cell and thereby impedes the MAVS-dependent production of type III IFNs ${ }^{93}$. However, further studies to elucidate the mechanistic details are still required.

Sequestration or relocalization of RLRs. To keep MDA5 sequestered and in an inactive state, the V proteins of several paramyxoviruses, including parainfluenza virus 5 (PIV5), directly bind to the helicase domain of MDA5 to block its ATPase activity ${ }^{6,94-96}$ (FIG. 3). VP35 from EBOV, NS1 from IAV, and the 4a protein from Middle East respiratory syndrome (MERS) coronavirus (MERS-CoV) target PACT, which inhibits the activation of RIG-I (and possibly MDA5) and thus prevents the production of IFNs ${ }^{97-99}$. In addition, the membrane protein $(\mathrm{M})$ from SARS-CoV, the $\mathrm{Z}$ protein from arenaviruses and the glycoprotein $(G)$ from human metapneumovirus (hMPV) bind to RIG-I to sequester it from downstream signalling molecules ${ }^{100-103}$.

Following the recognition of RNA ligands in the cytosol, RIG-I and MDA5 need to translocate to signalling-competent organelles, such as mitochondria, MAMs and peroxisomes, on which MAVS is localized. Recently, it was shown that the NS3 protein of DENV binds to the mitochondrial-targeting chaperone protein $14-3-3 \varepsilon$ to prevent the translocation of RIG-I to mitochondria that contain MAVS $^{104}$. Intriguingly, NS3 uses a highly conserved phosphomimetic ${ }^{64} \mathrm{RXEP}^{67}$ motif, which resembles a canonical phosphorylated serine-containing or threoninecontaining motif of cellular 14-3-3-binding proteins, to interact with $14-3-3 \varepsilon$ and impair RIG-I signalling and immune activation. A recombinant DENV encoding a mutant NS3 protein that is deficient in $14-3-3 \varepsilon$ binding is impaired in RIG-I antagonism and elicited an augmented production of IFN $\beta$, pro-inflammatory cytokines and ISGs, compared with wild-type DENV. The mutant DENV also induced a stronger activation of T cells. Interestingly, in contrast to the related NS3-NS4A protease complex of $\mathrm{HCV}$, which cleaves MAVS to block RIG-I signalling, NS2B-NS3 of DENV antagonizes RIG-I-dependent signalling in a proteolysis-independent manner.

Another strategy used by viruses to sequester RLRs is to relocalize them to other subcellular sites, often virus-induced structures. The nucleoprotein $(\mathrm{N})$ of RSV binds to MDA5 and relocalizes it (and also MAVS) to large viral inclusion bodies ${ }^{105}$, whereas the non-structural 


\section{Box 3 | Viral evasion of NLR-dependent immunity}

Several viruses target the NOD-like receptor (NLR)-mediated activation of the inflammasome. Non-structural protein 1 (NS1) from influenza A virus (IAV) inhibits the production of interleukin-1 $\beta$ (IL-1 $\beta$ ) and IL-18 during infection, although the molecular details remain elusive ${ }^{159}$. An IAV mutant with a partial deletion of the amino-terminal RNA-binding domain of NS1 induces the production of IL-1 $\beta$ and IL-18, which suggests that this domain is responsible for antagonizing the activation of caspase 1. Furthermore, PB1-F2 from IAV translocates into the mitochondrial inner membrane space to decrease mitochondrial membrane potential and to accelerate the fragmentation of mitochondria, thus blocking both the activation of the NOD-, LRRand pyrin domain-containing 3 (NLRP3) inflammasome and retinoic acid-inducible gene-I protein (RIG-I)-mitochondrial antiviral signalling protein (MAVS) signalling ${ }^{86}$. The $\mathrm{V}$ protein of measles virus (MeV) interacts with NLRP3 and relocalizes it to the perinuclear region following activation of the inflammasome, thereby inhibiting the secretion of IL- $1 \beta^{160}$. The M13L protein of myxoma virus and the vPOP protein of Shope fibroma virus bind to and target the adaptor protein apoptosis-associated speck-like protein containing a CARD (ASC) to prevent the activation of caspase 1 (REFS 161,162). Finally, the $\mathrm{F} 1 \mathrm{~L}$ protein of vaccinia virus and the ORF63 protein of Kaposi sarcomaassociated herpesvirus (KSHV) interact with NLRP1 to inhibit the activation of the inflammasome ${ }^{163,164}$, although it is unclear whether NLRP1 is directly involved in nucleic acid sensing. Interestingly, the ORF63 protein of KSHV is a viral homologue of NLRP1 and is able to interact with NLRP1, NLRP3 and nucleotide-binding oligomerization domain-containing protein 2 (NOD2), which suggests a broad antagonism of cellular NLRs.

protein in small segment (NSs) of severe fever with thrombocytopenia syndrome virus (SFTSV), a recently described phlebovirus in the Bunyaviridae family, relocalizes RIG-I and its upstream activator TRIM25 into cytoplasmic endosome-like structures, the formation of which is induced by infection with SFTSV ${ }^{106}$. Furthermore, stress granules are cytoplasmic bodies that have been proposed to act as antiviral platforms for $\mathrm{RLR}$ signalling, and the $3 \mathrm{C}^{\mathrm{pro}}$ of poliovirus and encephalomyocarditis virus (EMCV) cleave the cellular protein RAS GTPase-activating protein-binding protein 1 (G3BP1) to prevent the formation of RLR-containing stress granules ${ }^{107,108}$.

Finally, as NLR proteins also have a major role in virus sensing, several viruses have, in turn, evolved mechanisms to counteract NLR-mediated signalling pathways (BOX 3).

\section{Evasion of cGAS, IFI16 and STING signalling}

As cGAS and IFI16 are expressed in most host cells, all DNA viruses have to contend with these intracellular DNA sensors and escape immune signalling during both acute and persistent infection. In addition, retroviruses, which produce viral DNA during their life cycles, and other RNA viruses, inhibit cGAS and/or STING; however, in many cases the contribution of cGAS and/or STING antagonism to viral pathogenesis remains to be fully established.

Sequestration of viral genomes. The virions of HIV-1 carry a ssRNA genome, which is reverse-transcribed into DNA inside the infected host cell in preparation for chromosomal integration. As only a few copies of HIV-1 DNA integrate, the remaining copies are potentially vulnerable to detection by cytosolic sensors. It has been shown that HIV-1 uses the host $3^{\prime}$-repair exonuclease 1 (TREX1), the most abundant $3^{\prime}-5^{\prime}$ DNase in cells, to bind to and degrade excess cytosolic HIV-1 DNA ${ }^{109}$ (FIG. 4). The loss of TREX1 in host cells that are infected with HIV-1 led to an accumulation of HIV-1 reverse transcripts and triggered the production of IFN through STING, thereby preventing HIV-1 replication and spread. More recently, it has been reported that cGAS senses HIV-1 DNA and subsequently activates STING ${ }^{38}$, which suggests that the TREX1-mediated degradation of HIV-1 DNA enables the evasion of the sensor cGAS. Furthermore, two studies have shown that the capsid of HIV-1 (and also HIV-2) prevents the cGASdependent sensing of HIV complementary DNA (cDNA) by recruiting cellular proteins, such as cyclophilin A (CYPA) $)^{110,111}$; however, how CYPA prevents the activation of cGAS by HIV cDNA is unclear and remains to be determined.

Viral manipulation of STING post-translational modifications. HBV causes chronic hepatitis and increases the risk of developing liver cirrhosis and hepatocellular carcinoma. A recent study has shown that the reverse transcriptase and ribonuclease $\mathrm{H}$ domains of the $\mathrm{HBV}$ polymerase bind to STING to block its Lys63-linked ubiquitylation, thereby inhibiting the production of IFN $\beta^{112}$ (FIG. 4). The PLPs from human coronavirus NL63 (HCoV-NL63), SARS-CoV and porcine epidemic diarrhoea virus (PEDV), all of which are RNA viruses, have also been shown to associate with STING and block its dimerization and Lys63-linked ubiquitylation, thereby suppressing the activation of IRF3 (REFS 113-115). Therefore, SARS-CoV uses PLP to antagonize the Lys63-linked ubiquitylation of both RIG-I and STING (see above). Furthermore, it has been reported that the NS4B protein of HCV associates with STING, but the physiological relevance of this interaction remains to be fully elucidated ${ }^{116,117}$. A recent study has shown that the DNA tumour viruses human papillomavirus 18 (HPV18) and human adenovirus 5 (hAd5) inhibit the cGAS-STING pathway using their viral oncoproteins E7 and E1A, respectively ${ }^{118}$. E7 and E1A specifically bind to STING, but not to cGAS, although the authors did not study whether the dimerization and/or ubiquitylation of STING were affected by this interaction. Interestingly, the disruption of the cGAS-STING pathway was dependent on the LXCXE motif in the viral oncoproteins, which has previously been shown to be crucial for viral inhibition of the retinoblastoma tumour suppressor pathway. This finding suggests that the antagonism of innate immune signalling and the inactivation of tumour suppressor function share common mechanistic features. As many human DNA viruses encode proteins with LXCXE motifs, this study proposed that these viral proteins may represent a broad class of STING antagonists. Apart from the viral antagonism of STING dimerization and ubiquitylation, a recent study has shown that $\mathrm{KSHV}$, the causative agent of Kaposi sarcoma and several lymphoproliferative diseases in humans, encodes viral interferon regulatory factor 1 (vIRF1), which interacts with STING, thereby preventing TBK1 binding and TBK1-mediated phosphorylation of STING and STING activation $^{119}$. Notably, the authors screened more than 


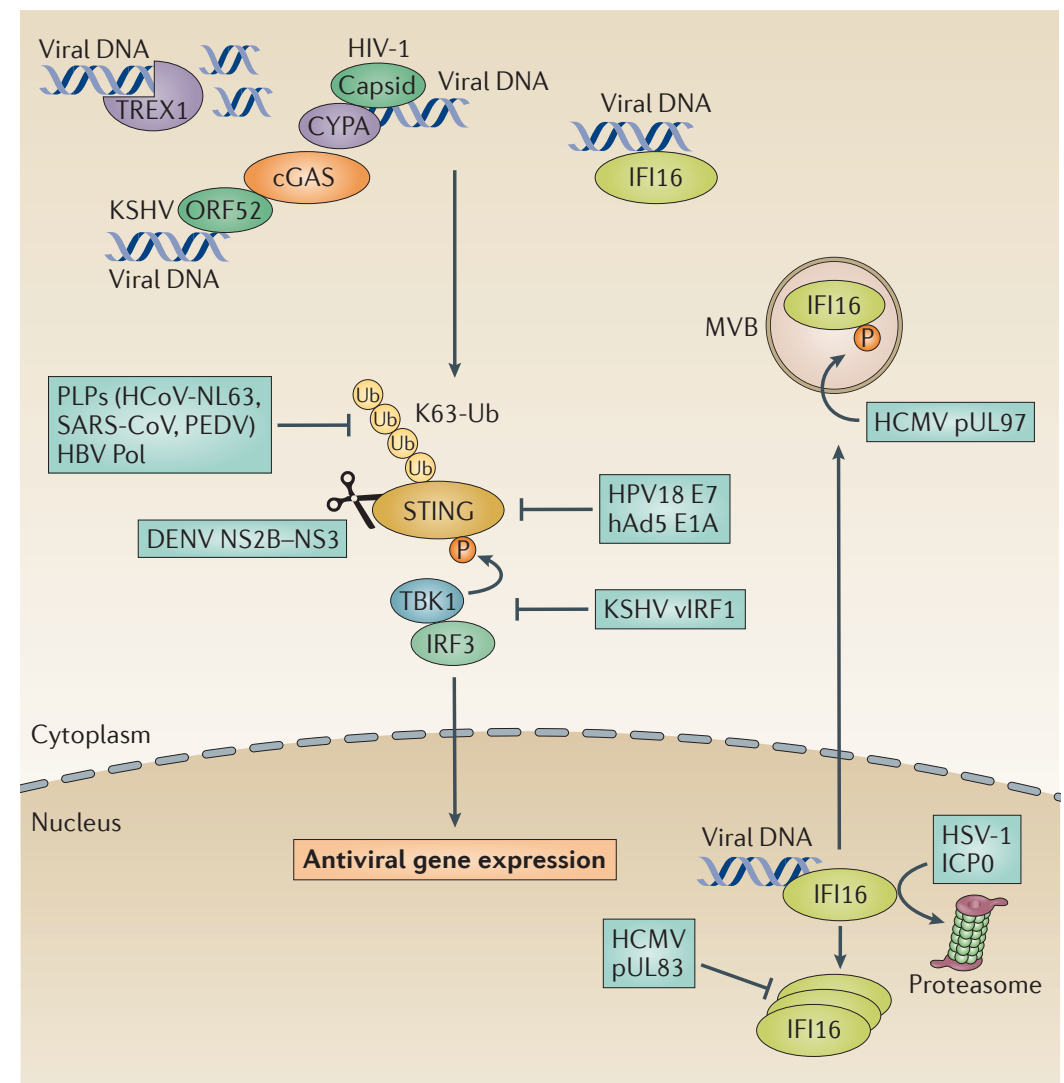

Figure 4 | Viral immune evasion of cGAS, IFI16 and STING. DNA viruses have developed molecular strategies to evade or inhibit intracellular DNA sensors and escape immune signalling during both acute and persistent infection. To prevent the activation of cyclic GMP-AMP synthase (cGAS), HIV-1 uses the cellular 3'-repair exonuclease 1 (TREX1) to degrade excess reverse transcribed viral DNA. In addition, the HIV-1 capsid recruits host-encoded factors, such as cyclophilin A (CYPA), which prevent the sensing of reverse transcribed DNA by cGAS. Furthermore, the tegument protein ORF52 of Kaposi sarcoma-associated herpesvirus (KSHV) binds to both viral DNA and cGAS to inhibit the activity of cGAS. To antagonize the activation of stimulator of interferon (IFN) genes (STING), the polymerase (Pol) of hepatitis B virus (HBV) and the papain-like proteases (PLPs) of human coronavirus NL63 (HCoV-NL63), severe acute respiratory syndrome (SARS)-associated coronavirus (SARS-CoV) and porcine epidemic diarrhoea virus (PEDV), prevent or remove the Lys63-linked ubiquitylation of STING. The viral IFN regulatory factor 1 (vIRF1) protein of KSHV blocks the TBK1-mediated phosphorylation of STING and thereby its signalling ability. The E7 protein of human papillomavirus 18 (HPV18) and the E1A protein of human adenovirus 5 (hAd5) bind to STING and inhibit its activation, whereas the NS2B-NS3 protease of dengue virus (DENV) cleaves STING to inactivate it. To inhibit IFN $\gamma$-inducible protein 16 (IFI16), infected cell protein 0 (ICP0) of herpes simplex virus 1 (HSV-1) targets IFI16 in the nucleus for proteasomal degradation, whereas the tegument protein pUL83 of human cytomegalovirus (HCMV) binds to IFI16 to prevent its oligomerization and thus its activation. The pUL97 protein of HCMV phosphorylates IFI16 during viral replication and relocalizes it from the nucleus to multivesicular bodies (MVBs). K63-Ub, Lys63-linked ubiquitylation; P, phosphate; Ub, ubiquitin.
Cleavage or degradation of DNA sensors and STING. As with most DNA viruses, HSV-1, which causes cold sores and can lead to encephalitis and keratitis, replicates in the nucleus and thus must counteract immune sensing in this cellular compartment. In agreement with this, infection with HSV-1 has been reported to trigger the proteasomal degradation of IFI16 in the nucleus (FIG. 4). A study has shown that HSV-1 mutants that lack the viral E3 ubiquitin ligase infected cell protein 0 (ICP0) or encode a catalytically inactive mutant of ICP0 failed to degrade IFI16, which suggests that ICP0 directly targets IFI16 for degradation ${ }^{121}$. In support of this, ICP0 binds to IFI16 and recruits it to ICP0-containing foci in the nucleus prior to degradation $^{121}$. However, the expression of ICP0 alone does not cause the degradation of IFI16 (REFS 122,123), and an ICP0-deficient HSV-1 mutant that was complemented with the human cytomegalovirus (HCMV) transactivating protein IE1 to promote viral gene expression was able to degrade IFI16 (REF. 122). These results indicate that ICP0 either directly targets IFI16 for degradation, or promotes the expression of another viral product that does so. With regard to STING, the protease complex NS2B-NS3 of DENV binds to and cleaves human STING to inactivate its function and to reduce the induction of IFNs ${ }^{124,125}$. By contrast, NS2B-NS3 fails to cleave mouse STING, which does not harbour the cleavage site found in human STING; this suggests that STING is a species-specific restriction factor of DENV replication. These findings, together with previous work that showed that the NS3-NS4A protease complex of the related HCV cleaves MAVS, highlight the convergence of DENV and HCV proteases on adaptors of DNA and RNA sensors (FIG. 4).

Sequestration or relocalization of DNA sensors. Recently, the tegument protein ORF52 of KSHV was shown to antagonize cGAS signalling ${ }^{126}$. ORF52 binds to both DNA and cGAS, thereby blocking the enzymatic activity of cGAS and decreasing the production of the second messenger cGAMP. Furthermore, the authors found that ORF52 homologues from other gammaherpesviruses, such as EBV and MHV68, also antagonized the activity of cGAS, which supports a conserved immune evasion function for ORF52. Together with vIRF1 from KSHV, which binds to and antagonizes STING, these findings demonstrate that gammaherpesviruses target both the sensor and adaptor proteins for efficient immune evasion. HCMV is a major cause of birth defects and is responsible for complications in patients who are immunosuppressed. HCMV targets IFI16 through a different mechanism than HSV-1 (FIG. 4). Although IFI16 is able to recognize HCMV DNA, the tegument protein pUL83 binds to the pyrin domain of IFI16, which prevents the oligomerization of IFI16 and subsequent immune signalling ${ }^{127}$. pUL83 uses its N terminus to bind to IFI16 and its $\mathrm{C}$ terminus to dissipate IFI16 oligomerization, which implies a concerted mechanism. Furthermore, the phosphorylation of Ser364 of pUL83 decreased its ability to antagonize IFI16, which suggests that host kinases can modulate the immunosuppressive 
activity of this viral protein. Notably, the potent ability of pUL83 to sequester IFI16 is probably owing to the fact that it is the most abundant virion component of HCMV, with more than 2,000 copies per mature virion ${ }^{128}$. More recently, a second complementary mechanism of IFI16 antagonism by HCMV has been proposed ${ }^{129}$. During HCMV replication, IFI16 translocates from the nucleus to the cytoplasm and mislocalizes to multivesicular bodies (MVBs), which prevents the detection of HCMV DNA in the nucleus. The authors found that the viral kinase pUL97 binds to and phosphorylates IFI16 and is required for IFI16 nuclear egress, which is consistent with the described role of pUL97 in the HCMV nuclear egress complex (FIC. 4). However, similar to the degradation of IFI16 by ICP0 from HSV-1, the introduction of pUL97 alone into cells did not trigger the translocation of IFI16, which suggests that other HCMV components are required. Interestingly, after translocating into MVBs, IFI16 seems to be incorporated into newly assembled HCMV virions, but the functional significance of this phenomenon has yet to be determined.

\section{Conclusion and outlook}

The past decade has seen rapid advances in our understanding of intracellular viral RNA and DNA sensors and how their activities are regulated. Fundamental principles that govern these sensors are emerging, such as the crucial roles of phosphorylation and the different forms of ubiquitylation in controlling the activation of PRRs, and some of the molecular features that distinguish 'non-self' PAMPs from host-derived nucleic acids. However, several gaps remain in our understanding of intracellular innate immune sensing. For example, RNA viruses and DNA viruses, such as DENV and HBV, antagonize both intracellular viral RNA and DNA receptors, indicating crosstalk between these two major sensing pathways, which remains poorly defined. Another conundrum concerns the spatial organization of individual intracellular sensors, and how they act in concert during an infection to elicit an effective host response. Furthermore, a better understanding of the physiological PAMPs that are recognized by intracellular pathogen receptors in an infected host cell would greatly aid in the design of immunostimulatory adjuvants or in prophylactic treatment.

In addition to the induction of IFNs and the activation of the inflammasome, the innate immune sensing of viral PAMPs can trigger autophagy ${ }^{130}$, and there is accumulating evidence that molecules that are involved in IFN-mediated innate immunity, such as cGAS, also have crucial roles in the regulation of this intracellular degradative process. It is essential to further our knowledge of how autophagy regulates immune and inflammatory responses so that this catabolic process can be harnessed for the development of antiviral therapeutics. Another exciting development in the field concerns the association of intracellular nucleic acid sensors with autoimmune diseases, such as Aicardi-Goutieres syndrome, SingletonMerten syndrome and systemic lupus erythematosus. As PRRs are able to mediate potent immune and inflammatory responses, dysregulation of their activity can trigger autoimmunity. This typically occurs in two ways: gain-of-function mutations that lead to the constitutive activation of innate immune sensors, or mutations in cellular nucleases that are responsible for eliminating nucleic acids that may stimulate intracellular PRRs ${ }^{131}$. Elucidating the mutations and mechanisms that are responsible for the aberrant activation of PRRs may inform therapeutic interventions for autoimmune diseases.

Numerous viral immune evasion mechanisms that specifically target intracellular PRRs have been uncovered, which highlight the importance of bypassing innate immune defence for viral replication and the common themes among strategies used by viruses. A key challenge is to translate this knowledge into useful applications for the development of new vaccines and antivirals. The ablation of specific viral PRR-antagonistic mechanisms while preserving other viral functions may give rise to live attenuated viruses, which are potential vaccine candidates. In particular, the elimination of several mechanisms of immune evasion from a viral pathogen may potently induce antiviral responses for long-lasting immunity, and also improve its safety profile. Similarly, the molecular mechanisms of viral immune escape may provide novel insights that may help to boost the immunogenicity of viral vectors for more effective vaccination. In addition, small-molecule inhibitors that target the interactions between viral proteins and crucial host immune molecules may act as potent antivirals and may increase the immune response of a patient. In certain cases in which an immune response is undesirable, such as gene therapy, it may be beneficial to use viral vectors with defined immune evasion mechanisms to prevent the early shutdown of transgene expression. In addition, new insights into the mechanisms by which overactive PRR signalling triggers inflammatory and/or autoimmune diseases could be useful for improving the immunogenicity of vaccines or reducing unwanted inflammation. Finally, the continuing emergence of viral variants that have increased virulence suggests that viruses can acquire novel strain-specific mechanisms of immune escape. Elucidating these strategies would shed light on viral evolution and provide molecular signatures for epidemiological monitoring.
1. Takeuchi, O. $\&$ Akira, S. Pattern recognition receptors and inflammation. Cell 140, 805-820 (2010).

2. Dambuza, I. M. \& Brown, G. D. C-type lectins in immunity: recent developments. Curr. Opin. Immunol. 32, 21-27 (2015)

3. Goubau, D., Deddouche, S. \& Reis e Sousa, C. Cytosolic sensing of viruses. Immunity 38, 855-869 (2013).

4. Schneider, W. M., Chevillotte, M. D. \& Rice, C. M Interferon-stimulated genes: a complex web of host defenses. Annu. Rev. Immunol. 32, 513-545 (2014).

5. Yoneyama, M et al The RNA helicase RIG-I has an essential function in double-stranded RNA-induced innate antiviral responses. Nat. Immunol. 5, 730-737 (2004)

6. Andrejeva, J. et al. The $\mathrm{V}$ proteins of paramyxoviruses bind the IFN-inducible RNA helicase, MDA5, and inhibit its activation of the IFN- $\beta$ promoter. Proc. NatI Acad. Sci. USA 101, 17264-17269 (2004).
7. Bruns, A. M. \& Horvath, C. M. LGP2 synergy with MDA5 in RLR-mediated RNA recognition and antiviral signaling. Cytokine 74, 198-206 (2015).

8. Kato, H. et al. Differential roles of MDA5 and RIG-I helicases in the recognition of RNA viruses. Nature 441, 101-105 (2006).

9. Loo, Y. M. et al. Distinct RIG-I and MDA5 signaling by RNA viruses in innate immunity. J. Virol. 82 , 335-345 (2008). 
10. Schlee, M. Master sensors of pathogenic RNA - RIG-I like receptors. Immunobiology 218, 1322-1335 (2013).

11. Rasmussen, S. B. et al. Herpes simplex virus infection is sensed by both Toll-like receptors and retinoic acidinducible gene-like receptors, which synergize to induce type I interferon production. J. Gen. Virol. 90, 74-78 (2009)

12. West, J. A. et al. An important role for mitochondrial antiviral signaling protein in the Kaposi's sarcomaassociated herpesvirus life cycle. J. Virol. $\mathbf{8 8}$ 5778-5787 (2014).

13. Chiang, J. J., Davis, M. E. \& Gack, M. U. Regulation of RIG-I-like receptor signaling by host and viral proteins. Cytokine Growth Factor Rev. 25, 491-505 (2014).

14. Gack, M. U., Nistal-Villan, E., Inn, K. S., Garcia-Sastre, A. \& Jung, J. U. Phosphorylationmediated negative regulation of RIG-I antiviral activity. J. Virol. 84, 3220-3229 (2010).

15. Maharaj, N. P., Wies, E., Stoll, A. \& Gack, M. U. Conventional protein kinase C- $\alpha($ PKC- $\alpha)$ and PKC- $\beta$ negatively regulate RIG-I antiviral signal transduction. J. Virol. 86, 1358-1371 (2012)

16. Wies, E. et al. Dephosphorylation of the RNA sensors RIG-I and MDA5 by the phosphatase PP1 is essential for innate immune signaling. Immunity 38, 437-449 (2013).

17. Sun, Z., Ren, H., Liu, Y., Teeling, J. L. \& Gu, J. Phosphorylation of RIG-I by casein kinase II inhibits its antiviral response. J. Virol. 85, 1036-1047 (2011).

18. Takashima, K., Oshiumi, H., Takaki, H. Matsumoto, M. \& Seya, T. RIOK3-mediated phosphorylation of MDA5 interferes with its assembly and attenuates the innate immune response. Cell Rep. 11, 192-200 (2015)

19. Kolakofsky, D., Kowalinski, E. \& Cusack, S. A structure-based model of RIG-I activation. RNA 18 2118-2127 (2012)

20. Kowalinski, E. et al. Structural basis for the activation of innate immune pattern-recognition receptor RIG-I by viral RNA. Cell 147, 423-435 (2011). This paper solves the crystal structures of auto-repressed RIG-I and activated RIG-I, showing that the cooperative binding of ATP and RNA to RIG-I frees the CARDs for downstream signalling.

21. Kok, K. H. et al. The double-stranded RNA-binding protein PACT functions as a cellular activator of RIG-I to facilitate innate antiviral response. Cell Host Microbe 9, 299-309 (2011).

22. Gack, M. U. et al. TRIM25 RING-finger E3 ubiquitin ligase is essential for RIG-I-mediated antiviral activity. Nature 446, 916-920 (2007)

This study identifies TRIM25 as a crucial activator of RIG-I, by inducing atypical Lys63-linked polyubiquitylation of the CARDs of RIG-I to promote antiviral signal transduction.

23. Oshiumi, H., Miyashita, M., Matsumoto, M $\Sigma$ Seya, T. A distinct role of Riplet-mediated K63-linked polyubiquitination of the RIG-I repressor domain in human antiviral innate immune responses. PLoS Pathog. 9, e1003533 (2013).

24. Horner, S. M., Liu, H. M., Park, H. S., Briley, J. \& Gale, M. Mitochondrial-associated endoplasmic reticulum membranes (MAM) form innate immune synapses and are targeted by hepatitis $C$ virus. Proc. Natl Acad. Sci. USA 108, 14590-14595 (2011).

25. Zeng, W. et al. Reconstitution of the RIG-I pathway reveals a signaling role of unanchored polyubiquitin chains in innate immunity. Cell 141, 315-330 (2010).

26. Liu, H. M. et al. The mitochondrial targeting chaperone $14-3-3 \varepsilon$ regulates a RIG-I translocon that mediates membrane association and innate antiviral immunity. Cell Host Microbe 11, 528-537 (2012).

27. Wu, B. et al. Structural basis for dsRNA recognition, filament formation, and antiviral signal activation by MDA5. Cell 152, 276-289 (2013).

28. Dixit, E. et al. Peroxisomes are signaling platforms for antiviral innate immunity. Cell 141, 668-681 (2010).

29. Hou, F. et al. MAVS forms functional prion-like aggregates to activate and propagate antiviral innate immune response. Cell 146, 448-461 (2011). The authors show that the activation of RIG-I-MAVS leads to the formation of large multiprotein complexes on mitochondria that are required for signal transduction, and that MAVS fibrils have prion-like characteristics.

30. Sato, S. et al. The RNA sensor RIG-I dually functions as an innate sensor and direct antiviral factor for hepatitis B virus. Immunity 42, 123-132 (2015).
31. Weber, M. et al. Influenza virus adaptation PB2-627K modulates nucleocapsid inhibition by the pathogen sensor RIG-I. Cell Host Microbe 17 309-319 (2015)

32. Yao, H. et al. ATP-dependent effector-like functions of RIG-I-like receptors. Mol. Cell 58, 541-548 (2015).

33. Dempsey, A. \& Bowie, A. G. Innate immune recognition of DNA: a recent history. Virology 479-480, 146-152 (2015)

34. Zhong, B. et al. The adaptor protein MITA links virus-sensing receptors to IRF3 transcription factor activation. Immunity 29, 538-550 (2008)

35. Ishikawa, H., Ma, Z. \& Barber, G. N. STING regulates intracellular DNA-mediated, type I interferondependent innate immunity. Nature 461, 788-792 (2009).

This work identifies STING as a crucial mediator of the type I IFN response to intracellular DNA species that are introduced by transfection, or that are derived from infection with DNA viruses or bacteria.

36. Sun, W. et al. ERIS, an endoplasmic reticulum IFN stimulator, activates innate immune signaling through dimerization. Proc. Natl Acad. Sci. USA 106 8653-8658 (2009).

37. Sun, L., Wu, J., Du, F., Chen, X. \& Chen, Z. J. Cyclic GMP-AMP synthase is a cytosolic DNA sensor that activates the type I interferon pathway. Science 339 , 786-791 (2013)

This paper demonstrates that cGAS is a key cytosolic DNA sensor that produces the second messenger cGAMP to activate the host IFN response.

38. Gao, D. et al. Cyclic GMP-AMP synthase is an innate immune sensor of HIV and other retroviruses. Science 341, 903-906 (2013).

39. Li, X. D. et al. Pivotal roles of CGAS-cGAMP signaling in antiviral defense and immune adjuvant effects. Science 341, 1390-1394 (2013).

40. Schoggins, J. W. et al. Pan-viral specificity of IFNinduced genes reveals new roles for cGAS in innate immunity. Nature 505, 691-695 (2014).

41. Wu, J. et al. Cyclic GMP-AMP is an endogenous second messenger in innate immune signaling by cytosolic DNA. Science 339, 826-830 (2013). The authors find that on cytosolic DNA stimulation, cGAMP acts as a second messenger by binding to STING to activate IRF3 and trigge the production of type I IFNs.

42. Gao, P. et al. Cyclic $\left[G\left(2^{\prime}, 5^{\prime}\right) \mathrm{pA}\left(3^{\prime}, 5^{\prime}\right) \mathrm{p}\right]$ is the metazoan second messenger produced by DNAactivated cyclic GMP-AMP synthase. Cell 153 1094-1107 (2013)

43. Ablasser, A. et al. cGAS produces a $2^{\prime}-5^{\prime}$-linked cyclic dinucleotide second messenger that activates STING. Nature 498, 380-384 (2013).

44. Ablasser, A. et al. Cell intrinsic immunity spreads to bystander cells via the intercellular transfer of cGAMP. Nature 503, 530-534 (2013).

This work demonstrates that cGAMP can be transferred between cells through gap junctions to confer antiviral immunity, which demonstrates a novel strategy used by the immune system.

45. Tsuchida, T. et al. The ubiquitin ligase TRIM56 regulates innate immune responses to intracellular double-stranded DNA. Immunity 33, 765-776 (2010)

46. Zhang, J., Hu, M. M., Wang, Y. Y. \& Shu, H. B. TRIM32 protein modulates type I interferon induction and cellular antiviral response by targeting MITA/STINC protein for K63-linked ubiquitination. J. Biol. Chem 287, 28646-28655 (2012).

47. Liu, S. et al. Phosphorylation of innate immune adaptor proteins MAVS, STING, and TRIF induces IRF3 activation. Science 347, aaa2630 (2015).

48. Tanaka, Y. \& Chen, Z. J. STING specifies IRF3 phosphorylation by TBK1 in the cytosolic DNA signaling pathway. Sci. Signal. 5, ra20 (2012)

49. Unterholzner, L. et al. IFI16 is an innate immune sensor for intracellular DNA. Nat. Immunol. 11, 997-1004 (2010)

The authors show that IFI 16 recruits STING on DNA stimulation to activate IRF3 and induce the production of IFNs.

50. Jin, T. et al. Structures of the HIN domain: DNA complexes reveal ligand binding and activation mechanisms of the AIM2 inflammasome and IFI16 receptor. Immunity 36, 561-571 (2012)

51. Bowie, A. G. \& Unterholzner, L. Viral evasion and subversion of pattern-recognition receptor signalling. Nat. Rev. Immunol. 8, 911-922 (2008).
52. Taylor, K. E. \& Mossman, K. L. Recent advances in understanding viral evasion of type I interferon. Immunology 138, 190-197 (2013).

53. Uchida, L. et al. The dengue virus conceals doublestranded RNA in the intracellular membrane to escape from an interferon response. Sci. Rep. 4, 7395 (2014).

54. Cardenas, W. B. et al. Ebola virus VP35 protein binds double-stranded RNA and inhibits $\alpha / \beta$-interferon production induced by RIG-I signaling. J. Virol. $\mathbf{8 0}$ 5168-5178 (2006)

55. Ramanan, P. et al. Structural basis for Marburg virus VP35-mediated immune evasion mechanisms. Proc Natl Acad. Sci. USA 109, 20661-20666 (2012).

56. Hatada, E. \& Fukuda, R. Binding of influenza A virus NS1 protein to dsRNA in vitro. J. Gen. Virol. 73 3325-3329 (1992).

57. Qian, X. Y., Chien, C. Y., Lu, Y., Montelione, G. T. \& Krug, R. M. An amino-terminal polypeptide fragment of the influenza virus NS1 protein possesses specific RNA-binding activity and largely helical backbone structure. RNA 1, 948-956 (1995).

58. Donelan, N. R., Basler, C. F. \& Garcia-Sastre, A. A recombinant influenza A virus expressing an RNAbinding-defective NS1 protein induces high levels of $\beta$-interferon and is attenuated in mice. J. Virol. 77 13257-13266 (2003)

59. Valentine, R. \& Smith, G. L. Inhibition of the RNA polymerase III-mediated dsDNA-sensing pathway of innate immunity by vaccinia virus protein E3. J. Gen Virol. 91, 2221-2229 (2010).

60. Bitko, V., Musiyenko, A., Bayfield, M. A., Maraia, R. J. $\&$ Barik, S. Cellular La protein shields nonsegmented negative-strand RNA viral leader RNA from RIG-I and enhances virus growth by diverse mechanisms. J. Virol. 82, 7977-7987 (2008).

61. Boonyaratanakornkit, J. et al. The $\mathrm{C}$ proteins of human parainfluenza virus type 1 limit double-stranded RNA accumulation that would otherwise trigger activation of MDA5 and protein kinase R. J. Virol. 85, 1495-1506 (2011)

62. Habjan, M. et al. Processing of genome $5^{\prime}$ termini as a strategy of negative-strand RNA viruses to avoid RIG-I-dependent interferon induction. PLOS ONE 3 , e2032 (2008)

63. Wang, H., Vaheri, A., Weber, F. \& Plyusnin, A. Old World hantaviruses do not produce detectable amounts of dsRNA in infected cells and the $5^{\prime}$ termini of their genomic RNAs are monophosphorylated. J. Gen. Virol. 92, 1199-1204 (2011).

64. Marq, J. B., Kolakofsky, D. \& Garcin, D. Unpaired 5'-ppp-nucleotides, as found in arenavirus doublestranded RNA panhandles, are not recognized by RIG-I. J. Biol. Chem. 285, 18208-18216 (2010)

65. Marq, J. B., Hausmann, S., Veillard, N., Kolakofsky, D. \& Garcin, D. Short double-stranded RNAs with an overhanging 5'-ppp-nucleotide, as found in arenavirus genomes, act as RIG-I decoys. J. Biol. Chem. 286, 6108-6116 (2011)

66. Hastie, K. M., Kimberlin, C. R., Zandonatti, M. A. MacRae, I. J. \& Saphire, E. O. Structure of the Lassa virus nucleoprotein reveals a dsRNA-specific 3'-to-5' exonuclease activity essential for immune suppression. Proc. Natl Acad. Sci. USA 108, 2396-2401 (2011).

67. Reynard, S., Russier, M., Fizet, A., Carnec, X $\&$ Baize, S. Exonuclease domain of the Lassa virus nucleoprotein is critical to avoid RIG-I signaling and to inhibit the innate immune response. J. Virol. 88, 13923-13927 (2014).

68. Gack, M. U. et al. Influenza A virus NS1 targets the ubiquitin ligase TRIM25 to evade recognition by the host viral RNA sensor RIG-I. Cell Host Microbe 5, 439-449 (2009).

This study identifies that the NS1 protein of influenza A virus binds to TRIM25 to inhibit the ubiquitylation and activation of RIG-I, which unveils a novel strategy used by influenza virus to escape host immunity.

69. Rajsbaum, R. et al. Species-specific inhibition of RIG-I ubiquitination and IFN induction by the influenza A virus NS1 protein. PLoS Pathog. 8, e1003059 (2012).

70. Inn, K. S. et al. Inhibition of RIG-I-mediated signaling by Kaposi's sarcoma-associated herpesvirus-encoded deubiquitinase ORF64. J. Virol. 85, 10899-10904 (2011)

71. Clementz, M. A. et al. Deubiquitinating and interferon antagonism activities of coronavirus papain-like proteases. J. Virol. 84, 4619-4629 (2010).

72. Wang, D. et al. The leader proteinase of foot-andmouth disease virus negatively regulates the type I interferon pathway by acting as a viral deubiquitinase. J. Virol. 85, 3758-3766 (2011). 
73. van Kasteren, P. B. et al. Deubiquitinase function of arterivirus papain-like protease 2 suppresses the innate immune response in infected host cells. Proc Natl Acad. Sci. USA 110, E838-E847 (2013).

74. Xu, C. et al. Downregulation of microRNA miR-526a by enterovirus inhibits RIG-I-dependent innate immune response. J. Virol. 88, 11356-11368 (2014).

75. Manokaran, G. et al. Dengue subgenomic RNA binds TRIM25 to inhibit interferon expression for epidemiological fitness. Science 350, 217-221 (2015).

76. Pauli, E. K. et al. The ubiquitin-specific protease USP15 promotes RIG-I-mediated antiviral signaling by deubiquitylating TRIM25. Sci. Signal. 7, ra3 (2014).

77. Davis, M. E. et al. Antagonism of the phosphatase PP1 by the measles virus $V$ protein is required for innate immune escape of MDA5. Cell Host Microbe 16, 19-30 (2014)

78. Mesman, A. W. et al. Measles virus suppresses RIG-I-like receptor activation in dendritic cells via DC-SIGN-mediated inhibition of PP1 phosphatases. Cell Host Microbe 16, 31-42 (2014).

79. Barral, P. M., Sarkar, D., Fisher, P. B. \& Racaniello, V. R. RIG-I is cleaved during picornavirus infection. Virology 391, 171-176 (2009).

80. Feng, Q. et al. Enterovirus $2 \mathrm{~A}^{\text {pro }}$ targets MDA5 and MAVS in infected cells. J. Virol. 88, 3369-3378 (2014).

81. Mukherjee, A. et al. The coxsackievirus B 3C protease cleaves MAVS and TRIF to attenuate host type I interferon and apoptotic signaling. PLoS Pathog. 7 e1001311 (2011)

82. Li, X. D., Sun, L., Seth, R. B., Pineda, G. \& Chen, Z. J. Hepatitis $C$ virus protease NS3/4A cleaves mitochondrial antiviral signaling protein off the mitochondria to evade innate immunity. Proc. Natl Acad. Sci. USA 102, 17717-17722 (2005).

83. Yang, Y. et al. Disruption of innate immunity due to mitochondrial targeting of a picornaviral protease precursor. Proc. Natl Acad. Sci. USA 104, 7253-7258 (2007).

84. Chen, Z. et al. GB virus B disrupts RIG-I signaling by NS3/4A-mediated cleavage of the adaptor protein MAVS. J. Virol. 81, 964-976 (2007).

85. Drahos, J. \& Racaniello, V. R. Cleavage of IPS-1 in cells infected with human rhinovirus. J. Virol. 83 11581-11587 (2009).

86. Yoshizumi, T. et al. Influenza A virus protein PB1-F2 translocates into mitochondria via Tom 40 channels and impairs innate immunity. Nat. Commun. 5, 4713 (2014).

This work demonstrates that influenza A virus accelerates mitochondrial fragmentation by reducing the mitochondrial inner membrane potential, thereby evading RIG-I signalling and the activation of the NOD-, LRR- and pyrin domain-containing 3 (NLRP3) inflammasome.

87. Varga, Z. T., Grant, A., Manicassamy, B. \& Palese, P. Influenza virus protein PB1-F2 inhibits the induction of type I interferon by binding to MAVS and decreasing mitochondrial membrane potential. J. Virol. 86 8359-8366 (2012).

88. Wei, C. et al. The hepatitis B virus X protein disrupts innate immunity by downregulating mitochondrial antiviral signaling protein. J. Immunol. 185 1158-1168 (2010)

89. Barral, P. M. et al. MDA-5 is cleaved in poliovirusinfected cells. J. Virol. 81, 3677-3684 (2007).

90. Xia, M. et al. Mitophagy enhances oncolytic measles virus replication by mitigating DDX58/RIG-I-like receptor signaling. J. Virol. 88, 5152-5164 (2014).

91. Shi, C. S. et al. SARS-coronavirus open reading frame-9b suppresses innate immunity by targeting mitochondria and the MAVS/TRAF3/TRAF6 signalosome. J. Immunol. 193, 3080-3089 (2014).

92. Goswami, R. et al. Viral degradasome hijacks mitochondria to suppress innate immunity. Cell Res. 23, 1025-1042 (2013)

93. You, J. et al. Flavivirus infection impairs peroxisome biogenesis and early antiviral signaling. J. Virol. 89 12349-12361 (2015).

94. Rodriguez, K. R. \& Horvath, C. M. Amino acid requirements for MDA5 and LGP2 recognition by paramyxovirus $\mathrm{V}$ proteins: a single arginine distinguishes MDA5 from RIG-I. J. Virol. 87, 2974-2978 (2013).

95. Motz, C. et al. Paramyxovirus V proteins disrupt the fold of the RNA sensor MDA5 to inhibit antiviral signaling. Science 339, 690-693 (2013). The authors find that $\mathrm{V}$ proteins from paramyxoviruses directly bind to the innate immune sensor MDA5 to unfold its ATP-hydrolysis domain, which prevents its activation.
96. Childs, K. S., Andrejeva, J., Randall, R. E. $\&$ Goodbourn, S. Mechanism of MDA5 inhibition by paramyxovirus V proteins. J. Virol. 83, 1465-1473 (2009)

97. Luthra, P. et al. Mutual antagonism between the Ebola virus VP35 protein and the RIG-I activator PACT determines infection outcome. Cell Host Microbe 14, 74-84 (2013).

98. Tawaratsumida, K. et al. Quantitative proteomic analysis of the influenza A virus nonstructural proteins NS1 and NS2 during natural cell infection identifies PACT as an NS1 target protein and antiviral host factor. J. Virol. 88, 9038-9048 (2014).

99. Siu, K. L. et al. Middle east respiratory syndrome coronavirus 4 a protein is a double-stranded RNA binding protein that suppresses PACT-induced activation of RIG-I and MDA5 in the innate antiviral response. J. Virol. 88, 4866-4876 (2014).

100. Siu, K. L. et al. Severe acute respiratory syndrome coronavirus $\mathrm{M}$ protein inhibits type I interferon production by impeding the formation of TRAF3 TANK-TBK $1 /$ IKKE complex. J. Biol. Chem. 284, 16202-16209 (2009).

101. Fan, L., Briese, T. \& Lipkin, W. I. Z proteins of New World arenaviruses bind RIG-I and interfere with type interferon induction $J$ Virol 84, 1785-1791 (2010).

102. Xing, J., Ly, H. \& Liang, Y. The Z proteins of pathogenic but not nonpathogenic arenaviruses inhibit RIG-l-like receptor-dependent interferon production. J. Virol. 89, 2944-2955 (2015).

103. Bao, X. et al. Human metapneumovirus glycoprotein $\mathrm{G}$ inhibits innate immune responses. PLoS Pathog. 4, e 1000077 (2008)

104. Chan, Y. K. \& Gack, M. U. A phosphomimetic-based mechanism of dengue virus to antagonize innate immunity. Nat. Immunol. 17, 523-530 (2016). This study reveals that the NS3 protein of DENV binds to $14-3-3 \varepsilon$ to prevent RIG-I translocation to mitochondria, thereby inhibiting IFN induction. The authors also find that a phosphomimetic RxEP motif in NS3 is crucial for $14-3-3 \varepsilon$ binding, suggesting viral mimicry of cellular phosphorylated binding motifs.

105. Lifland, A. W. et al. Human respiratory syncytial virus nucleoprotein and inclusion bodies antagonize the innate immune response mediated by MDA5 and MAVS. J. Virol. 86, 8245-8258 (2012)

106. Santiago, F. W. et al. Hijacking of RIG-I signaling proteins into virus-induced cytoplasmic structures correlates with the inhibition of type I interferon responses. J. Virol. 88, 4572-4585 (2014).

107. Ng, C. S. et al. Encephalomyocarditis virus disrupts stress granules, the critical platform for triggering antiviral innate immune responses. J. Virol. 87 9511-9522 (2013)

108. White, J. P., Cardenas, A. M., Marissen, W. E. \& Lloyd, R. E. Inhibition of cytoplasmic mRNA stress granule formation by a viral proteinase. Cell Host Microbe 2, 295-305 (2007).

109. Yan, N., Regalado-Magdos, A. D. Stiggelbout, B. Lee-Kirsch, M. A. \& Lieberman, J. The cytosolic exonuclease TREX 1 inhibits the innate immune response to human immunodeficiency virus type 1 . Nat. Immunol. 11, 1005-1013 (2010).

110. Rasaiyaah, J. et al. HIV-1 evades innate immune recognition through specific cofactor recruitment. Nature 503, 402-405 (2013).

This work highlights the importance of evading innate immune recognition by shielding viral genomes with the host proteins cleavage and polyadenylation specificity factor 6 (CPSF6) and cyclophilins.

111. Lahaye, X. et al. The capsids of HIV-1 and HIV-2 determine immune detection of the viral cDNA by the innate sensor cGAS in dendritic cells. Immunity 39 1132-1142 (2013)

112. Liu, Y. et al. Hepatitis B virus polymerase disrupts K63-linked ubiquitination of STING to block innate cytosolic DNA-sensing pathways. J. Virol. 89, 2287-2300 (2015)

113. Chen, X. et al. SARS coronavirus papain-like protease inhibits the type I interferon signaling pathway through interaction with the STING-TRAF3-TBK complex. Protein Cell 5, 369-381 (2014).

114. Sun, L. et al. Coronavirus papain-like proteases negatively regulate antiviral innate immune response through disruption of STING-mediated signaling. PLOS ONE 7, e30802 (2012)

115. Xing, Y. et al. The papain-like protease of porcine epidemic diarrhea virus negatively regulates type interferon pathway by acting as a viral deubiquitinase. J. Gen. Virol. 94, 1554-1567 (2013).
116. Nitta, S. et al. Hepatitis C virus NS4B protein targets STING and abrogates RIG-I-mediated type I interferondependent innate immunity. Hepatology 57, 46-58 (2013)

117. Ding, Q. et al. Hepatitis C virus NS4B blocks the interaction of STING and TBK 1 to evade host innate immunity. J. Hepatol 59, 52-58 (2013).

118. Lau, L., Gray, E. E., Brunette, R. L. \& Stetson, D. B. DNA tumor virus oncogenes antagonize the cGASSTING DNA sensing pathway. Science 350, 568-571 (2015).

This study shows that the oncoproteins of certain DNA viruses are potent inhibitors of DNA sensors, which suggests that some viral oncogenes have evolved dual function during the co-evolution of host and virus.

119. Ma, Z. et al. Modulation of the CGAS-STING DNA sensing pathway by gammaherpesviruses. Proc. Natl Acad. Sci. USA 112, E4306-E4315 (2015).

120. Sun, C. et al. Evasion of innate cytosolic DNA sensing by a gammaherpesvirus facilitates establishment of latent infection. J. Immunol. 194, 1819-1831 (2015).

121. Orzalli, M. H., DeLuca, N. A. \& Knipe, D. M. Nuclear IFI1 6 induction of IRF-3 signaling during herpesviral infection and degradation of IFI 16 by the viral ICPO protein. Proc. Natl Acad. Sci. USA 109, E3008-E3017 (2012)

122. Cuchet-Lourenco, D., Anderson, G., Sloan, E., Orr, A. $\&$ Everett, R. D. The viral ubiquitin ligase ICPO is neither sufficient nor necessary for degradation of the cellular DNA sensor IFI1 6 during herpes simplex virus 1 infection. J. Virol. 87, 13422-13432 (2013).

123. Diner, B. A., Lum, K. K., Javitt, A. \& Cristea, I. M Interactions of the antiviral factor interferon $\gamma$-inducible protein 16 (IFI16) mediate immune signaling and herpes simplex virus- 1 immunosuppression. Mol. Cell Proteomics 14, 2341-2356 (2015)

124. Aguirre, S. et al. DENV inhibits type I IFN production in infected cells by cleaving human STING. PLoS Pathog. 8, e1002934 (2012).

125. Yu, C. Y. et al. Dengue virus targets the adaptor protein MITA to subvert host innate immunity. PLoS Pathog. 8 e1002780 (2012)

126. Wu, J. J. et al. Inhibition of cGAS DNA sensing by a herpesvirus virion protein. Cell Host Microbe 18 333-344 (2015)

This study identifies that ORF52 of KSHV binds to DNA and also cGAS to block the enzymatic activity of cGAS, which facilitates the evasion of the host IFN response.

127. Li, T., Chen, J. \& Cristea, I. M. Human cytomegalovirus tegument protein pUL83 inhibits IFI16-mediated DNA sensing for immune evasion. Cell Host Microbe 14 591-599 (2013)

128. Varnum, S. M. et al. Identification of proteins in human cytomegalovirus (HCMV) particles: the HCMV proteome. J. Virol. 78, 10960-10966 (2004)

129. Dell'Oste, V et al. Innate nuclear sensor IFI16 translocates into the cytoplasm during the early stage of in vitro human cytomegalovirus infection and is entrapped in the egressing virions during the late stage. J. Virol. 88, 6970-6982 (2014).

30. Deretic, V., Saitoh, T. \& Akira, S. Autophagy in infection, inflammation and immunity. Nat. Rev. Immunol. 13, 722-737 (2013)

131. Kato, H. \& Fujita, T. RIG--like receptors and autoimmune diseases. Curr. Opin. Immunol. 37 40-45 (2015).

132. Dalet, A. Gatti, E. \& Pierre, P. Integration of PKRdependent translation inhibition with innate immunity is required for a coordinated anti-viral response. FEBS Lett. 589, 1539-1545 (2015).

133. Lloyd, R. E. Regulation of stress granules and P-bodies during RNA virus infection. Wiley Interdiscip Rev. RNA 4, 317-331 (2013)

134. Hornung, V., Hartmann, R., Ablasser, A. $\Sigma$ Hopfner, K. P. OAS proteins and cGAS: unifying concepts in sensing and responding to cytosolic nucleic acids. Nat. Rev. Immunol. 14, 521-528 (2014)

135. Malathi, K., Dong, B., Gale, M. \& Silverman, R. H. Smal self-RNA generated by RNase $L$ amplifies antiviral innate immunity. Nature 448, 816-819 (2007).

136. Oshiumi, H. et al. DDX60 is involved in RIG-I-dependent and independent antiviral responses, and its function is attenuated by virus-induced EGFR activation. Cell Rep. 11, 1193-1207 (2015).

137. Hornung, V. et al. AIM2 recognizes cytosolic dsDNA and forms a caspase-1-activating inflammasome with ASC. Nature 458, 514-518 (2009). 
138. Rathinam, V. A. et al. The AIM2 inflammasome is essential for host defense against cytosolic bacteria and DNA viruses. Nat. Immunol. 11, 395-402 (2010)

139. Wen, H., Miao, E. A. \& Ting, J. P. Mechanisms of NOD like receptor-associated inflammasome activation. Immunity 39, 432-441 (2013)

140. Kanneganti, T. D. Central roles of NLRs and inflammasomes in viral infection. Nat. Rev. Immunol. 10, 688-698 (2010).

141. Sabbah, A. et al. Activation of innate immune antiviral responses by NOD2. Nat. Immunol. 10, 1073-1080 (2009).

142. Allen, I. C. et al. The NLRP3 inflammasome mediates in vivo innate immunity to influenza $A$ virus through recognition of viral RNA. Immunity 30, 556-565 (2009).

143. Ichinohe, T., Lee, H. K., Ogura, Y., Flavell, R $\&$ Iwasaki, A. Inflammasome recognition of influenza virus is essential for adaptive immune responses. J. Exp. Med. 206, 79-87 (2009).

144. Poeck, H. et al. Recognition of RNA virus by RIG-I results in activation of CARD9 and inflammasome signaling for interleukin $1 \beta$ production. Nat. Immunol. 11, 63-69 (2010).

145. Hornung, V. et al. 5'-triphosphate RNA is the ligand for RIG-I. Science 314, 994-997 (2006).

146. Pichlmair, A. et al. RIG-I-mediated antiviral responses to single-stranded RNA bearing 5'-phosphates. Science 314, 997-1001 (2006)

This paper demonstrates that single-stranded RNA bearing 5'-phosphates is a RIG-I ligand, which enables RIG-I to distinguish between 'self' and 'non-self'.

147. Goubau, D. et al. Antiviral immunity via RIG-I-mediated recognition of RNA bearing 5'-diphosphates. Nature 514, 372-375 (2014).

148. Saito, T., Owen, D. M., Jiang, F., Marcotrigiano, J. $\&$ Gale, M. Innate immunity induced by composition- dependent RIG-I recognition of hepatitis C virus RNA. Nature 454, 523-527 (2008).

149. Uzri, D. \& Gehrke, L. Nucleotide sequences and modifications that determine RIG-I/RNA binding and signaling activities. J. Virol. 83, 4174-4184 (2009).

150. Baum, A., Sachidanandam, R. \& Garcia-Sastre, A Preference of RIG-I for short viral RNA molecules in infected cells revealed by next-generation sequencing. Proc. Natl Acad. Sci. USA 107, 16303-16308 (2010).

151. Rehwinkel, J. et al. RIG-I detects viral genomic RNA during negative-strand RNA virus infection. Cell 140, 397-408 (2010).

152. Pichlmair, A. et al. Activation of MDA5 requires higherorder RNA structures generated during virus infection. J. Virol. 83, 10761-10769 (2009).

153. Zust, R. et al. Ribose 2'-O-methylation provides a molecular signature for the distinction of self and nonself mRNA dependent on the RNA sensor MDA5 Nat. Immunol. 12, 137-143 (2011).

154. Herzner, A. M. et al. Sequence-specific activation of the DNA sensor cGAS by $Y$-form DNA structures as found in primary HIV-1 CDNA. Nat. Immunol. 16, 1025-1033 (2015).

155. Orzalli, M. H. \& Knipe, D. M. Cellular sensing of viral DNA and viral evasion mechanisms. Annu. Rev. Microbiol. 68, 477-492 (2014).

156. Orzalli, M. H. et al. cGAS-mediated stabilization of IFI 16 promotes innate signaling during herpes simplex virus infection. Proc. Natl Acad. Sci. USA 112, E1773-E1781 (2015).

157. Muruve, D. A. et al. The inflammasome recognizes cytosolic microbial and host DNA and triggers an innate immune response. Nature 452, 103-107 (2008).

158. Kanneganti, T. D. et al. Critical role for Cryopyrin/Nalp3 in activation of caspase- 1 in response to viral infection and double-stranded RNA. J. Biol. Chem. 281, 36560-36568 (2006).
159. Stasakova, J. et al. Influenza A mutant viruses with altered NS1 protein function provoke caspase- 1 activation in primary human macrophages, resulting in fast apoptosis and release of high levels of interleukins $1 \beta$ and 18. J. Gen. Virol. 86, 185-195 (2005).

160. Komune, N., Ichinohe, T., Ito, M. \& Yanagi, Y. Measles virus $V$ protein inhibits NLRP3 inflammasomemediated interleukin-1 $\beta$ secretion. J. Virol. 85 13019-13026 (2011).

161. Johnston, J. B. et al. A poxvirus-encoded pyrin domain protein interacts with ASC-1 to inhibit host inflammatory and apoptotic responses to infection. Immunity 23, 587-598 (2005).

162. Dorfleutner, A et al. A Shope fibroma virus PYRINonly protein modulates the host immune response. Virus Genes 35, 685-694 (2007).

163. Gerlic, M. et al. Vaccinia virus F1L protein promotes virulence by inhibiting inflammasome activation. Proc. Natl Acad. Sci. USA 110, 7808-7813 (2013)

164. Gregory, S. M. et al. Discovery of a viral NLR homolog that inhibits the inflammasome. Science 331, 330-334 (2011).

This paper shows that ORF63 of KSHV is a viral homologue of the receptor NLRP1 and subverts the function of NLRP1 and several other NLR members.

\section{Acknowledgements}

The authors apologize to all those colleagues whose important contributions could not be cited owing to space constraints. Current research in the Gack laboratory is supported by the US National Institutes of Health (grants AI087846 and AI118509) and a grant from the Progressive Multifocal Leukoencephalopathy (PML) Consortium.

Competing interests statement

The authors declare no competing interests. 